\title{
Katalog der in den Sammlungen des Senckenberg Deutschen Entomologischen Instituts aufbewahrten Typen - Coleoptera: Cerambycidae
}

\author{
Hannelore Gaedike ${ }^{1}$ und Lutz Behne ${ }^{2}$ \\ ${ }^{1}$ Florusstraße 5, 53225 Bonn \\ ${ }^{2}$ Senckenberg Deutsches Entomologisches Institut, Eberswalder Straße 90, 15374 Müncheberg \\ Published on 2013-06-14
}

\section{Zusammenfassung}

Die Typen der Familie Cerambycidae (Coleoptera) in den Sammlungen des Senckenberg Deutschen Entomogischen Instituts werden aufgelistet. Mitgeteilt werden jeweils das Zitat der Erstbeschreibung und Angaben über das vorhandene Typenmaterial. Die Sammlungen enthalten 445 Primärtypen, davon 78 Holotypen, 29 Lectotypen und Syntypen von 338 Arten. Von 57 weiteren Arten existieren Paratypen oder Paralectotypen. Von 25 Arten sind als Typen etikettierte Tiere wahrscheinlich nicht beschrieben.

\section{Summary}

The type specimens of the family Cerambycidae (Coleoptera) in the collections of the Senckenberg Deutsches Entomologisches Institut are listed. In each case, the original description is cited and information on the extant type material is presented. The collections contain 445 primary types, including 78 holotypes, 29 lectotypes and syntypes of 338 species. Paratypes and paralectotypes of a further 57 species are present. Specimens of 25 species are labelled as types, but these species have probably not been described.

\section{Cerambycidae}

abakumovi sarkandicum DANILEVSKY, 2004, Dorcadion Les Cahiers Magellanes 36: 4

o Holotypus, Kazakhstan, north foothills of Dzhungarsky Alatau, 10 km SW Sarkand, 900-1100 m, 14.V.2002, leg. \& Coll. Danilevsky; 122 Paratypen siehe Originalbeschreibung.

SDEI: 2 ○ $0^{\star}$ Paratypus, Kazakhstan, Dzhungarsky Alatau, Sarkand, 900 m, 14.V.2002, leg. M. DANILEVSKY.

accola HEYDEN, 1894, Dorcadion

Dtsch. ent. Ztschr. 38, 87

o Holotypus, Asia minor, Mardin, in Coll. v. Heyden, im SDEI.

\section{achilleae HolzSCHuH, 1971, Phytoecia}

Mitt. Forstl. Bundes-Versuchsanstalt Wien 94, 68-69

Holotypus, 47 Paratypen, Anatolien, Amanusgebirge, Prov. Adana, Nurdğai geçidi, 1000-1200 m, 13.-27.V. 1970, an Achillea, leg. Holzschuh und 15., 16.V.1969, leg. Wewalka, in Coll. Holzschuh.

SDEI: 1 Paratypus, Anatolien, Prov. Adana, Nurdag geçidi, 13.-27.V.1970, 800-1300 m, leg. HolzschuH.

aculeatus meridionalis HÜDEPOHL, 1979, Oxymerus

Ent. Arb. Mus. G. Frey 28, 23-25, 28

o Holotypus, Argentinien, Chaco de Santiago del Estero, Bords du Rio Salado, Env. d' Icano, 1900, leg. WAgner, 
im Museum Paris; zahlreiche o ${ }^{\star}$ ㅇ Paratypen siehe Originalbeschreibung.

SDEI: 1 Paratypus, Paraguay, Concepcion, leg. WeIske; 1 Paratypus, Paraguay.

acutivittis KraAtz, 1879, Clytus

Dtsch. ent. Ztschr. 23, 111-112, Taf. 1, Fig. 1

Mehrere Ex. Ost-Sibirien, Amur, leg. Christoph; 1 Ex. Japan, leg. v. Hilgendorf, im Königlichen Museum (Museum für Naturkunde Berlin), Syntypen.

SDEI: 4 Syntypen, Sibirien, Amur, leg. Christoph, Coll. KraAtz, Coll. F. Müller, Coll. v. Heyden.

\section{acutoides SchWARZER, 1925, Glenea}

Ent. Bl. 21, 151

Mehrere Syntypen, Formosa, Kosempo, 7.VI.1912, leg. SAUTER.

SDEI: 3 Syntypen, Formosa, Kosempo, 7.VI.1912, leg. SAUTER.

\section{aestuans ubangiensis FIEDLER, 1938, Ceroplesis}

Dtsch. ent. Ztschr. 1938, 592

Holotypus, 25 Paratypen, Afrika, Fort Sibut, Fort Crampel, Surango, Njam-Njam, Uganda, im Museum Berlin, Museum Brüssel und SDEI.

SDEI: 1 Paratypus, Nyam-Nyam, Coll. KraAtz.

\section{albicrus AuriviLuIus, 1907, Phrosyne}

Dtsch. ent. Ztschr. 1907, 77-78

Mehrere Syntypen, Kamerun, leg. ConRadT, via SchenKLING an AURIVILLIUS, im SDEI.

SDEI: 5 Syntypen, Kamerun, leg. Conradt, Coll. KRAATZ.

\section{albifrons HeYDEN, 1886, Menesia}

Dtsch. ent. Ztschr. 30, 276-277

Holotypus, Amur, Suyfun-Fluss, 1885, leg. Dörries, durch Staudinger, No. 366, in Coll. v. Heyden, im SDEI.

\section{albivitttis KraATz, 1879, Liopus}

Dtsch. ent. Ztschr. 23, 112-113, Taf. 1, Fig. 9

Holotypus, Ost-Sibirien, Amur, leg. Christoph, im SDEI 1877.

\section{albocinctus Melzer, 1930, Atrypanius}

Arch. Inst. biol. S. Paulo 3, 198-199, Taf. 17, Fig. 16

Mehrere Syntypen; Estado de Sao Paulo, Capital, Santos, Guaraja, leg. Melzer; Amparo, leg. Navarro DE ANDRADE; Estado de Santa Catharina, Joinville, leg. Schmith; Estado de Minas Geraes, Mar de Hespanha, leg. Zikan; Estado Epirito Santo, leg. Zikan.

SDEI: 1 Syntypus, Est. S. Paulo, Guaraja, 9.XII.1920, leg. Melzer.

albomaculatus SCHWARZER, 1924, Pseudonephelotus Treubia 5, 237
Mehrere Syntypen, Süd-Sumatra, Wai Lima, Lampongs, XI-XII.1921, leg. Karny \& Siebers.

SDEI: 1 Syntypus, mit den Angaben der Beschreibung, ex Museum Buitenzorg, No. 160.

\section{alboscutellata KRAATz, 1862, Stenostola}

Berl. ent. Ztschr. 6, 124-125

$o^{\star}+$ o Syntypen, Griechenland (?), leg. KRÜPER.

SDEI: 1 Paralectotypus, ohne Fundortangaben, Designation: SAMA.

albovittigera HeYDEN, 1863, Phytoecia

Berl. ent. Ztschr. 7, 130

Mehrere Syntypen, europäische Türkei, durch FEHR, in Coll. v. Heyden.

SDEI: Lectotypus, Turcia, durch Fehr, Coll. v. Heyden, Designation: SAMA.

algerica DesBrochers, 1870, Phytoecia

Abeille 7, 126-127

3 Syntypen, Algérie (Bône), in Coll. Desbrochers.

SDEI: 1 hist. Ex. „Phytoecia algerica DEsBR. Al.“, nach SAMA „forse Paratypus“.

\section{alienus Melzer, 1932, Probatius}

Rev. Ent. S. Paulo 2, 431-432, Taf. 6, Fig. 4

Brasilien, Estado de Minas Geraes, Passa Quatro, Fazenda dos Campos, leg. Zikan, Holotypus, mehrere Paratypen, in Coll. Melzer und im SDEI.

SDEI: 2 Paratypen, Brasilien, Minas, Passa Quatro, Faz. dos Campos, leg. ZikAN.

altaica PlaVILSTSHIKOV, 1933, Agapanthia

Ent. Nachr. bl. Troppau 7, 113-116

Mehrere Syntypen, Sibiria, Altai centr., Ojratia, pag. Shebalino, 27.VI., 1.-21.VII.1932, leg. VALDAEv.

SDEI: 3 Syntypen, Altai centr., Shebalino, 24., 27.VI., 3.VII.1932, leg. VALDAEV.

\section{altaicum Suvorov, 1909, Neodorcadion}

Rev. Russe d'Ent. 9, 89-90

Mehrere Syntypen, Altai, Anfang VII.; in der Nähe des Dorfes Altaiskaja, Anfang VI.; in der Nähe des Dorfes Bolschenarymskaja, 1906; leg. JaCobson, Coll. SEmeNOV-TIAN-SHANSKY.

SDEI: 2 Syntypen, Altai, Altaiskaja, 3.VII.1906, leg. JaCOBSON, Coll. KLÄGER.

\section{alternans ScHWARZER, 1925, Saimia}

Ent. Bl. 21, 62

Mehrere Syntypen, Formosa: Kankau; Kosempo, X.1912; Pilam, VIII.1912; leg. SAUTER.

SDEI: Formosa, leg. SAuter: 11 Ex. Kosempo, VI.1909/ 7.VII.1909/ 7.VII.1911/ 7.X.1911/ 7.VIII.1911/ 22.V. 1912/ VI.1912; 2 Ex. Kankau (Koshun), V., IX.1912, Syntypen. 
alticollis KRAATZ, 1879, Grammoptera

Dtsch. ent. Ztschr. 23, 103-104

Holotypus, Ost-Sibirien, Amur, leg. Christoph, im SDEI, Coll. KraAtz.

amurense KrAATz, 1879, Asemum

Dtsch. ent. Ztschr. 23, 97

Mehrere Syntypen, Ost-Sibirien, Amur, leg. CHrIsTOPH.

SDEI: 5 Syntypen, Amur, 1877, leg. Christoph, Coll. KraAtZ.

amurensis KRAATz, 1879, Agapanthia

Dtsch. ent. Ztschr. 23, 115-116

Mehrere Syntypen, Ost-Sibirien, Amur, leg. CHrisTOPH.

SDEI: Lectotypus, Sibirien, Amur, leg. Christoph, Coll. KraAtz, Designation: Sama.

amurensis KraAtz, 1879, Pachyta

Dtsch. ent. Ztschr. 23, 69-71, Taf. 1, Fig. 21-23

Mehrere Syntypen, Ost-Sibirien, Amur, leg. CHrIsTOPH.

SDEI: 7 Syntypen, Sibirien, Amur, 1877, leg. Christoph, Coll. KraAtz, Coll. F. Müller, Coll. v. Heyden.

amurensis KRAATZ, 1879, Toxotus

Dtsch. ent. Ztschr. 23, 100-101

Mehrere Syntypen, Ost-Sibirien, Amur, leg. CHrisTOPH.

SDEI: 5 Syntypen, Sibirien, Amur, leg. Christoph, Coll. KraAtz, Coll. v. Heyden.

anancyloides SchWARZER, 1925, Coptops

Ent. Bl. 21, 61

Mehrere Syntypen, Formosa: Fuhosho, VI.1909; Hoozan, IX.1910; leg. SAUTER.

SDEI: Syntypen, Formosa, leg. SAUTer: 3 Ex. Fuhosho, VI., VII.09; 2 Ex. Hoozan, 7.IX.1908 und 1910.

androsovi Suvorov, 1909, Compsodorcadion

Rev. Russe d'Ent. 9, 93-94

Mehrere Syntypen; in der Nähe der Station Kara-Tscha-

kat (kleine Barsuki-Steppe), Gebiet Turgai, Anfang IV.1898; in den Sand-Steppen Dzhideti, Gebiet Turgai, 7.-12.V.1904, leg. DubJANSKy; Umgebung der Station Tshelkar (grosse Barsuki-Steppe), IV.1908, leg. ANDrosov.

SDEI: $2 \sigma^{\star}$ Syntypen, Kirghisien, Tshakat, distr., Steppe Basuki, Gebiet Turgai, leg. Androsov, Coll. KlägER (als ab. rufiscapus Suv. bezettelt).

angolensis LePeSme \& BREUning, 1955, Hypoeschrus Bull. Soc. ent. Fr. 60, 45

o Holotypus, Angola, Benguella, leg. Wellmann, Coll. KrAATZ, im SDEI. angulicollis HEYDEN, 1878, Strangalia

In: SchneIder \& Leder, Beitr. Kauk. Käferf., 323-324

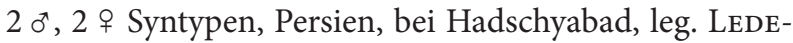
RER, in Coll. TÜRK.

SDEI: Lectotypus, Hadschyabad, leg. LEDERER, durch TÜRK, von SAMA festgelegt.

\section{angustata Melzer, 1932, Oreodera}

Rev. Ent. S. Paulo 2, 423-424, Taf. 6, Fig. 2

Brasilien, Estado de Pernambuco, Tapéra, leg. PiCKel; Estado do Espirito Santo, leg. Hoffmann; Holotypus, mehrere Paratypen, in Coll. Melzer und im SDEI.

SDEI: 1 Paratypus, Espir. Santo, X.1920-II.1921, leg. HOFFMANN.

angustefasciatus HeYDEN, 1884, Anaglyptus

Dtsch. ent. Ztschr. 28, 297-298

Holotypus, Insel Askold, Vladivostok, 1883, in Coll. v. Heyden, im SDEI (durch Koltze).

angustisternis GouneLLE, 1911, Euryptera

Ann. Soc. ent. Fr. 80, 9-10

15 ơ, 18 + Syntypen, Brasilien, Prov. Goyaz, Jatahy.

SDEI: 2 Syntypen, Brésil, Prov. Goyaz, Jatahy, Coll. KRAATZ.

anticemaculatus SCHWARZER, 1925, Chlorophorus

Ent. Bl. 21, 27-28

Mehrere Syntypen, Formosa: Alikang, VI.1909; Kankau, V., VI., VIII.1912; leg. SAUTER.

SDEI: Formosa, leg. SAuter, Syntypen: 3 Ex. Kankau, Koshun, V., VI., VIII.1912; 1 Ex. Alikang, VI.1909.

anulicornis SCHWARZER, 1925, Glenea

Ent. Bl. 21, 149-150

Mehrere Syntypen, Formosa: Kosempo, VI.1911; Kankau, VII.1912; leg. SAUTER.

SDEI: Formosa, leg. SAUter, Syntypen: 1 Ex. Kosempo, VI.1911; 1 Ex. Kankau, Koshun, VII.1912.

apicale SCHWARZER, 1925, Iphra?

Ent. Bl. 21, 23

Mehrere Syntypen, Formosa: Fuhosho, 7.IX.; Kankau, 7.IV., IX.1912; leg. SAUTER.

SDEI: Formosa, leg. SAUter, Syntypen: 3 Ex. Kankau, IV., V., IX.1912; 1 Ex. Fuhosho, 7.IX.

apicalis AuRIVILLIUS, 1907, Euniolia

Dtsch. ent. Ztschr. 1907, 80

Mehrere Syntypen, Kamerun, leg. Conradt, im SDEI. SDEI: 1 Syntypus, Kamerun, leg. ConradT, Coll. KraAtz.

\section{apicalis SCHWARzen, 1924, Pterolophia}

Treubia 5, 238

Mehrere Syntypen, Süd-Sumatra, Wai Lima, Lampongs, XI.-XII.1921, leg. Karny \& Siebers.

SDEI: 1 Syntypus, mit den Angaben der Beschreibung. 
apicalis GounelLe, 1911, Stenygra

Ann. Soc. ent. Fr. 80, 123

4 ơ, 2 ㅇ Syntypen, Brasilien, Prov. Goyaz, Jatahy, Santa Cathrina, Espirito-Santo, Minas Geraës, im Britischen Museum, SDEI und in Coll. GounelLe.

SDEI: 1 Syntypus, Brasilien, Coll. KraAtz.

\section{apicalis SCHWARzER, 1924, Tetraommatus}

\section{Treubia 5, 236}

Mehrere Syntypen, Süd-Sumatra, Wai Lima, Lampongs, XI.-XII.1921, leg. Karny \& Siebers.

SDEI: 1 Syntypus, mit den Angaben der Beschreibung, No. 17, ex Museum Buitenzorg.

\section{arandae Schramm, 1911, Dorcadion}

Bol. Real. Soc. Esp. Hist. Nat. 11, 306-307

$3 o^{*}, 5$ ㅇ Syntypen, Spanien, Sierra de Cameros, Logroño, leg. ARANDA, im Museum Madrid.

SDEI: 5 Syntypen, Sierra de Cameros, Logrono, leg. Aranda, Coll. Kläger, Coll. v. Heyden.

\section{araneipes SCHWARZER, 1925, Raphuma}

Ent. Bl. 21, 28-29

Holotypus, Formosa, Kankau, IV.1912, leg. SAUTER, im SDEI (als gracilipedes bezettelt!).

\section{araneipes pallida SCHWARZER, 1925, Raphuma}

Ent. Bl. 21, 29

Holotypus, Formosa, Kosempo, 22.V.1912, leg. SAUTER, im SDEI (als gracilipedes pallida bezettelt!).

ardoisi Schramm, 1909, Dorcadion

Bol. Real. Soc. Esp. Hist. Nat. 9, 296-298

Mehrere Syntypen, Spanien, Sierra de Gredos, 1900.

SDEI: 2 Syntypen, Sierra de Gredos, Avila, V.1904, leg. Schramm, Coll. v. Heyden.

\section{argonauta Suvorov, 1913, Dorcadion}

Rev. Russe d'Ent. 13, 74-75

Mehrere Syntypen, im Gouv. Eriwan, Umgebung vom Dorfe Kisilkom, leg. Maljuschenko und DobrowLJANSKY.

SDEI: 17 Syntypen, Eriwan G., Kisilkom, leg. MaljusCHENKo, Coll. KLÄGER (davon 8 Ex. ab. pallescens Suv.).

arietinum charynense DANILEVSKY, 1996 Dorcadion

Lambillionea 96: 610-611 (charynensis lapsus)

o Holotypus, Kazakhstan, north-east Turaigyr Mts near Charyn river, $1200 \mathrm{~m}$, leg. \& Coll. Danilevsky; 330 Paratypen vom gleichen Fundort in der Sammlung des Autors.

SDEI: $10^{\top}, 1$ क 9 Patatypen, Kazakhstan, Charyn Riv., 1200 m, 3.V.1994, leg. M. DANILEvSKY.

arietinum zhalanash DANILEVSKY, 1996 Dorcadion Lambillionea 96: 614 o Holotypus, 641 Paratypen, Kazakhstan, 26 km N Zhalanash, 1300 m, 15.IV.1991, leg. \& Coll. M. Danilevsky SDEI: $10^{\star}, 1$ ㅇ Paratypen, vom gleichen Fundort.

armata var. nigricornis StIERLIN, 1864, Strangalia

Berl. ent. Ztschr. 8, 153

o Syntypen, Sizilien, durch Pfister.

SDEI: $1 \sigma^{7}$, Sicil., Designation: SAma (Die ursprünglich vorgenommene Auszeichnung als Holotypus ist als Lectotypenfestlegung zu werten).

ascoldensis HEYDEN, 1884, Atimura

Dtsch. ent. Ztschr. 28, 298

2 Syntypen, Insel Askold, in Coll. v. Heyden und Coll. Koltze, im SDEI, Amur, 1884.

asiaticum SCHWARZER, 1925, Neosphaerion

Ent. B1. 21, 22

Mehrere Syntypen, Formosa: Kosempo, IX., X.1911/ V., VI., VII.1912; Fuhosho, 7.IX.1912; Sokutsu, VII.1912; leg. SAUTER.

SDEI: Formosa, leg. SAUter, Syntypen: 1 Ex. Fuhosho, 7.IX.; 1 Ex. Kosempo, 22.V.1912; 3 Ex. Banshoryo-Distr., Sokutsu, 22.VI., 7.VII.1912.

\section{assimilis AurVILLIUs, 1908, Pelargoderus}

Dtsch. ent. Ztschr. 1908, 215-216

Mehrere Syntypen, Neu-Pommern, Herbertshöhe, NeuLauenburg, leg. v. BeNNIGSEN.

SDEI: 2 Syntypen, D. N. Guinea, Herbertshöhe, NeuLauenburg, Coll. v. Bennigsen.

\section{atticum KraAtZ, 1873, Dorcadion}

In: Küster, Käf. Europ. 29, 72; als var. von saulcy Tномs. beschrieben

12 o Syntypen, ohne Fundort, durch v. Heldreich und KRÜPER.

SDEI: 5 Syntypen, ohne Fundort, Coll. KraAtz.

\section{auratipennis KoLBE, 1900, Mecothyrea}

Berl. ent. Ztschr. 45, 302-303

$1 \sigma^{\prime}$, West-Afrika, Mhonda; 1 , Kilimandscharo, leg. v. BeNNIGSEn; Syntypen.

SDEI: 1 Syntypus, Kilimandscharo, Coll. v. BenNigsen.

aureovittata AuRIVILLIUS, 1907, Sophronica

Dtsch. ent. Ztschr. 1907, 81-82

Mehrere Syntypen, Kamerun, leg. ConRADT, im SDEI.

SDEI: 1 Syntypus, Kamerun, leg. Conradt, Coll. KraAtz.

auricomum rubescens M. SCHMIDT, 1924, Callichroma Dtsch. ent. Ztschr. 1924, 320

Mehrere Syntypen, Ecuador, Gualaquiza, Coll. B. SchwARZER.

SDEI: 1 Syntypus, Columbien, Cauca. 
auricomum viridescens M. ScHMIDT, 1924, Callichroma Dtsch. ent. Ztschr. 1924, 320

Mehrere Syntypen, Brasilien, Para, Tapajos (n. Bat. 1870), Obidos (Hoffmanns Stett.); Rio Jurua, Rio Purus (Moeckel und Duhaut Bln.); Columbien (Pehlke, Stett., Coll. Schw.), Esmeralda (Da.); ohne Fundortangabe, ex Coll. Richter Bln.

SDEI: 4 Syntypen, Columbia, Esmeralda, Coll. Horn.

\section{aurivilliusi HeLLER, 1912, Paracomeron}

Ent. Mitt. 1, 174-175, Fig. 4

Mehrere Syntypen, Nova Guinea, Coll. v. Bennigsen. SDEI: 1 Syntypus, Neuguinea, leg. und Coll. v. BenNigSEN.

\section{aurovittatum KrAATZ, 1873, Dorcadion}

In: Küster, Käf. Europ. 29, 81

Mehrere Syntypen, Klein-Asien, durch Kindermann, in Coll. Mniszeck und Coll. KraAtz.

SDEI: 1 Syntypus, Asia minor, Coll. KraATz.

australis SCHWARZER, 1926, Gnoma

Senckenbergiana 8, 286-287, Taf. 4, Fig. 26, 27

$\sigma^{\star}$ Holotypus, $20^{\star}, 3$ ㅇ Paratypen, Coen Distrikt, Cape York, leg. Hacker, im SDEI, im Senckenberg-Museum und in Coll. SCHWARZER.

SDEI: 4 Paratypen, Queensland, Coen Distrikt, Cape York, leg. HACKer, Coll. HACKER.

\section{balcanica HAMPE, 1870, Pachyta}

Berl. ent. Ztschr. 14, 336

5 Syntypen, Balkan, leg. Haberhauer.

SDEI: 3 Syntypen, Balkan, leg. Haberhauer, Coll. KraAtz, Coll. v. Heyden.

\section{bangi HEYDEN, 1894, Dorcadion}

Dtsch. ent. Ztschr. 38, 89, Taf. 1, Fig. 4

4 Syntypen, Asia minor, Kastemuni, durch Staudinger. SDEI: 2 Syntypen, Kastemuni, durch STAudinger, Coll. KrAATZ.

\section{basale KrAATz, 1873, Dorcadion}

In: Küster, Käf. Europ. 29, 86

ơ Holotypus, Armenien, leg. Steven, in Coll. KraAtz, im SDEI.

basalis Auriviluius, 1908, Abryna

Dtsch. ent. Ztschr. 1908, 221-222

+ Holotypus, Salomonen, Insel Tulagi, leg. v. Bennigsen, im SDEI.

basalis JORDAN, 1894, Frea

Novit. Zool. 1, 233-234

Mehrere Syntypen, Kuilu, 1892, leg. Mocquerys und LOANDA.

SDEI: 1 Syntypus, Loanda, Coll. KraATz. basalis AuniviLluus, 1908, Pterolophia

Dtsch. ent. Ztschr. 1908, 222

† Holotypus, Salomonen, Insel Tulagi, leg. v. BENnigsen, im SDEI.

basalis SCHWARZER, 1925, Xylotrechus

Ent. Bl. 21, 25-26

Mehrere Syntypen, Formosa: Taihorinsho, 7.IX., VI.1911; Alikang, VI.1909; Kosempo, VI.1909/ 7.XII.1911; Fuhosho, VI.1909; Kankau, V.1912; leg. SAUTER.

SDEI: Formosa, leg. SAUter, Syntypen: 2 Ex. Kosempo, 7.IX.1909/1912; 4 Ex. Kankau, Koshun, IV., VI.1912; 3 Ex. Taihorin, 22.VI., 22.VIII., VI.1911 (nicht Taihorinsho); 2 Ex. Alikang, VI.1909.

\section{beckeri KraAtz, 1873, Dorcadion}

In: Küster, Käfer Europ. 29, 71

$10^{\top}, 1$ i Syntypen, Derbent, leg. Becker.

SDEI: 1 Syntypus, Derbent, Coll. KraAtz.

bennigseni KoLBE, 1897, Afrocomis

Die Käfer Dtsch. Ost-Afr., 352

Mehrere Syntypen; Khutu, Lumba-Thal, 28.XI.1894, leg. Stuhlmann; Usaramo, leg. v. Bennigsen; Sansibar (wohl Festland, leg. C. W. Schmidt).

SDEI: 1 Syntypus, Usaramo, Coll. v. Bennigsen.

bennigseni AuriviLlius, 1908, Docohammus

Dtsch. ent. Ztschr. 1908, 217, Taf. 3, Fig. 3

Holotypus, D. O. Afrika, Mpuapua, leg. v. Bennigsen, im SDEI, Coll. v. BenNigsen.

bennigseni AurVILLIUs, 1908, Monochamus

Dtsch. ent. Ztschr. 1908, 216-217

Mehrere Syntypen, Karolinen-Inseln, Ponape, leg. v. BeNNIGSEN.

SDEI: 13 Syntypen, Karolinen, Ponape, Coll. v. BennIGSEN.

bennigseni AuriviLluus, 1908, Memophas

Dtsch. ent. Ztschr. 1908, 215

† Holotypus, Deutsch Neu-Guinea, Herbertshöhe, leg. v. Bennigsen; im SDEI, Coll. v. Bennigsen.

bennigseni KoLBE, 1897, Plocederus

Die Käfer Dtsch. Ost-Afr., 297

Mehrere Syntypen, Dar-es-Salaam, VIII.1894, leg. STUHLMANN und leg. v. BENNIGSEN.

SDEI: 1 Syntypus, Dar-es-Salaam, leg. v. Bennigsen, Coll. v. BenNigsen.

\section{bennigseni AURIVILLIUS, 1908, Rosenbergia}

Dtsch. ent. Ztschr. 1908, 217-218

Mehrere Syntypen, Deutsch Neu-Guinea, Sattelberg, leg. v. BENNIGSEN.

SDEI: 1 Syntypus, D. N. Guinea, Sattelberg. 
bennigseni LAMEERE, 1912, Xaurus

Mém. Soc. Ent. Belg. 21, 161-162

1 , Holländ. Neu-Guinea, Wendesi, leg. Meyere, im Museum Amsterdam; 1 i , Deutsch-Neu-Guinea, leg. v. Bennigsen, durch Horn; $10^{*}$, Grenze zwischen Holländ. und Deutsch-Neu-Guinea, Coll. SchenkLING, im SDEI: Syntypen.

SDEI: $10^{\star}, 1$ ㅇ Syntypen, D. N. Guinea, Deutsch-Holländ. Grenze, Coll. v. Bennigsen (nicht Coll. SchenkLIng!).

biapiculata AurIVILLIUS, 1908, Dictamnia

Dtsch. ent. Ztschr. 1908, 211

+ Holotypus, Neu-Pommern, Herbertshöhe, Coll. v. BENNIGSEN, im SDEI.

\section{bicallosus KraAtz, 1882, Clytus}

Dtsch. ent. Ztschr. 26, 307, 335-336

Holotypus, Samarkand, leg. Haberhauer.

SDEI: Holotypus, Samarkand, Stdgr. 81.

\section{bicolor GounELLE, 1911, Euryptera}

Ann. Soc. ent. Fr. 80, 7-9

$6 o^{\star}, 9$ ๆ Syntypen, Brasilien, Prov. Goyaz, Jatahy.

SDEI: 2 Syntypen, Brésil, Prov. Goyaz, Jatahy, Coll. KrAATZ.

\section{bicolor SCHWARZER, 1924, Glenea}

Nova Guinea 15, 60

Mehrere Syntypen, N. West Neu-Guinea: Prauwenbivak, Idenburg River, IX.1920; Pionierbivak, Mamberamo River, XII.1920; leg. v. Heurn.

SDEI: 1 Syntypus, N. Guinea Exped., Idenburg River, IX.1920, Pionierbivak, leg. v. Heurn, ex Museum Buitenzorg.

\section{bicolor SCHWARZER, 1924, Hoplosyllitus}

Nova Guinea 15, 58

Mehrere Syntypen, N. West New-Guinea, Pionierbivak, Mamberamo River, 1.VIII.1920, leg. v. HeurN, holländ. Grenze.

SDEI: 1 Syntypus, mit den Angaben der Beschreibung, ex Museum Buitenzorg.

\section{bicolor Kratiz, 1862, Obrium}

Berl. ent. Ztschr. 6, 126

Mehrere Syntypen, Griechenland, leg. KRÜPER.

SDEI: Lectotypus, Graecia, leg. KrüPer, Coll. KraAtZ; 1 Paralectotypus, ohne Fundortangaben, Designation: SAMA.

\section{bicolor HeYDEN, 1886, Pidonia}

Dtsch. ent. Ztschr. 1886, 276

Holotypus, Amur, Suyfun, in Coll. v. Heyden, im SDEI, 1885, leg. DöRrIEs.

bienerti HeYDEN, 1885, Polyarthron

Dtsch. ent. Ztschr. 29, 311-312, Taf. 4, Fig. 1
2 Syntypen, Persien, leg. BienerT.

SDEI: 1 Syntypus, Persien, leg. BienerT.

bifarius HEYDEN, 1889, Clytus

Dtsch. ent. Ztschr. 1889, 329-330

Holotypus, Asia minor, Hadjin, durch Staudinger, im SDEI, Coll. v. HeYden.

\section{bifascianus SCHWARZER, 1925, Eoporimimus}

\section{Ent. B1. 21, 147}

Mehrere Syntypen, Formosa: Fuhosho, 7.VII., 7.VIII., 7.IX.; Sokutsu, 7.VII.1912; leg. SAUTER.

SDEI: Formosa, leg. Sauter, Syntypen: 8 Ex. Fuhosho, 7.VII., 7.VIII., 7.IX.; 2 Ex. Banshoryo-Distr., Sokutsu, 22.VI., 7.VII.1912.

\section{bifasciatus KraAtz, 1879, Rhopoloscelis}

Dtsch. ent. Ztschr. 1879, 113-114, Taf. 1, Fig. 10

o Holotypus, Ost-Sibirien, Amur, leg. Christoph, im SDEI, Coll. KraATZ, 1877.

biforme Kratz, 1873, Dorcadion

In: Küster, Käf. Europ. 29, 53

Mehrere Syntypen, Persien, bei Hadschgabad, leg. CHrIsTOPH.

SDEI: 6 Syntypen, Hadschgabad, Coll. KraATz.

\section{bimaculicollis SCHWARzER, 1925, Chlorophorus}

Ent. Bl. 21, 28

Mehrere Syntypen, Formosa: Fuhosho, 7.IX.; Kosempo, 7.VIII.1911; leg. SAUTER.

SDEI: Formosa, leg. SAUter, Syntypen: 1 Ex. Fuhosho, 7.IX.; 1 Ex. Kosempo, 7.VIII.1911.

\section{biocellatus ScHWARzer, 1925, Olenecamptus}

Ent. Bl. 21, 63

Mehrere Syntypen, Formosa, Kankau, IV., V., VI., VII., IX.1912, leg. SAUTER.

SDEI: 6 Syntypen, Formosa, Kankau, Koshun, V., VI., VII., IX.1912, leg. SAUTER.

\section{bipunctithorax BREUnING, 1958, Glenea}

Beitr. Ent. 8, 493

+ Holotypus, Neu Guinea, Mamberano River, Pionierbivak, 1920, leg. v. Heurn, im SDEI.

bivittata AuriviluIUs, 1907, Nupserha

Dtsch. ent. Ztschr. 1907, 83

Mehrere Syntypen, Kamerun, leg. ConRAdT, im SDEI. SDEI: 1 Syntypus, Kamerun, leg. Conradt, Coll. KraATZ.

bivittata Salto, 1980, Pidonia

Kontyû, Tokyo 48 (2), 291-294, Fig. 1-6

$0^{\star}$ Holotypus, Pilu-shenmu (near Mt. Pilushan, $2.440 \mathrm{~m}$ alt.), Hwalien Hsien, 8.V.1977, leg. SAIto; $2090^{\star}$, 135 + Paratypen, dito, VI.1977 und 1978, leg. FujITA, Itoh, Kuboki, NiIsato, Oda, Sakaino, Schimomura 
und Saito; im National Science Museum (Nat. Hist.), Tokyo; in Coll. SaIto und andere Coll.

SDEI: 2 Paratypen, Taiwan, Hwalien Hsien (Shen-mu), 8.V.1977, leg. SAIto.

\section{bizonatus Gounelle, 1911, Rhinotragus}

Ann. Soc. ent. Fr. 80, 15-16

3 ơ, 11 i Syntypen, Brasilien, Prov. Goyaz, Jatahy.

SDEI: 2 Syntypen, Brésil, Prov. Goyaz, Jatahy, Coll. KraAtZ.

\section{bodemeyeri ReITTER, 1903, Callidium}

Wien. ent. Ztg. 22, 46

$10^{\star}$ Syntypus, Kleinasien, Bulghar-Dagh, leg. v. BodEMEYER.

SDEI: als Paralectotypus, Kleinasien, Bulghar-Maaden, Designation: SAMA.

\section{bodemeyeri DANIEL, 1900, Dorcadion}

Soc. Ent. 15, 140

Mehrere Syntypen, Asia minor, leg. Escherich und leg. v. BODEMEYER.

SDEI: 10 Syntypen, Asia minor, Eski-Chéhir, leg. v. BoDEmeyer, Coll. KraAtz und Coll. v. Heyden.

bodemeyeri ReITTER, 1913, Phytoecia

Dtsch. ent. Ztschr. 1913, 665-666

Mehrere Syntypen, Persien, Luristan, durch V. BodEMEYER.

SDEI: 1 Syntypus, Persien, Luristan, durch v. BodEMEyer, Coll. v. Heyden.

boisduvali maculosa Schwarzer, 1929, Batocera

Senckenbergiana 11, 367, Fig. 21

+ Holotypus, mehrere Paratypen, Neu-Guinea, Senckenberg-Museum (Col. 79).

SDEI: 1 Paratypus, D. N. Guinea, Herbertshöhe, Coll. v. BenNigsen.

bothridera Lameere, 1903, Macrotoma

Mem. Soc. Ent. Belg. 11, 103

Mehrere Syntypen: Süd Madagascar, Plateau de l` Androy, im Musée Paris; Mahabo, Andrahomana, leg. Alluaud, im Musée de Trig.

SDEI: $1 \sigma^{\star}, 1$ ㅇ Paralectotypen, Plateau de l'Androy-Reg. d'Ambovombe, Coll. KraAtz; Designation: Quentin \& VILLIERS.

\section{bougainvillei LAMEERE, 1912, Hastertia}

Mem. Soc. Ent. Belg. 21, 163

$1 \sigma^{7}, 2$ ㅇ, iles Salomon, ile Bougainville, durch Hastert DE Luxembourg; 2 ㅇ, Kieta, im SDEI: Syntypen.

SDEI: 2 Syntypen, D. Salomons Ins., Kieta, Coll. KraATz.

brachyptera Daniel, 1899, Acmaeops

Col. Stud. 2, 73
Mehrere Syntypen, Turkestan, Musart am Nordfluss des Tian-Schan, durch HAuser.

SDEI: 1 Syntypus, Thian-Schan, Musart, VI.1894, Coll. Hauser-Coll. F. Müller.

\section{brannani ScHaUFUSS, 1870, Dorcadion}

Nunq. otiosus 1, 64

Mehrere Syntypen, Lusitania, Mont. Estrellensis, 18.VI. 1867, $5000 \mathrm{ft}$., leg. Schaufuss.

SDEI: 4 Syntypen, Lusitania, Sierra de Estrella, leg. W. Schaufuss, Coll. L. W. Schaufuss.

\section{bulbifera KoLBE, 1894, Phrystola}

Stett. ent. Ztg. 55, 40

Mehrere Syntypen, Kamerun, im Gebirge bei Buea, leg. Preuss.

SDEI: 1 Syntypus, Kamerun, Gebirge Buea, 17.X.1891, leg. Preuss, Coll. KraAtz.

\section{bytinskii HeYROvSkY, 1954, Clytus}

Ent. Arb. Mus. Frey 5, 395-396

Holotypus, Paratypen (3 o $0^{\star}, 2$ ), Israel, Rehobot, IV.1948, leg. Bytinski-Salz, in Coll. Bytinski-Salz und Coll. HEYROVSKY.

SDEI: 1 Paratypus, mit den Angaben der Beschreibung, V.1948 (nicht IV!).

\section{campestre GouneLLE, 1909, Octoplon}

Ann. Soc. ent. Fr. 77, 667

Mehrere Syntypen, Brasilien, Prov. Goyaz, Jatahy, Minas Bahia, leg. GounELLE.

SDEI: 3 Syntypen, Brésil, Prov. Goyaz, Jatahy, Coll. KRAATZ.

campestris GounELLE, 1909, Eburodacrys

Ann. Soc. ent. Fr. 77, 633-634, Fig. 19

Mehrere Syntypen, Brasilien, Prov. Goyaz, Jatahy, Bahia, Minas Geraes, Cidade de Condeuba, Sertao de Diamantina.

SDEI: 2 Syntypen, Brésil, Prov. Goyaz, Jatahy, Coll. KRAATZ.

carinicollis ScHWARzER, 1925, Nortia

Ent. Bl. 21, 20-21

Mehrere Syntypen, Formosa: Fuhoshu, V.1909; Kosempo, XII.1911/ VI.1912; Sokutsu, V.1911/ VI.1912/ VII.1912; leg. SAUTER.

SDEI: Formosa, leg. SAuter, Syntypen: 2 Ex. Fuhosho, V.1909/ 7.IX.; 2 Ex. Banshoryo Distr., Sokutsu, 7.VI., 7.VII.1912; 3 Ex. Kosempo, 7.VII.1911/ 7.VI.1912.

caroli Leprieur, 1876, Plocaederus

Bull. Soc. ent. Fr. (5) 6, VIII

Syntypen, Algerien, LePrieur.

SDEI: 1 Paralectotypus, Bou-Saada, LEPRIEUR, Designation: SAMA. 
catalai VILLIERS, 1939, Leucographus

Bull. Soc. ent. Fr. 44, 206-207, 208, Fig. 1

${ }^{\top}$ Holotypus, mehrere Paratypen, Madagaskar, Prov. d'Ankarampotsy und Tantamala, Region Fianarantsoa an der Ostküste, im Museum Paris.

SDEI: 1 Paratypus, Madagaskar, Foret Cote Est.

\section{cervae FrIVALDSZKY, 1892, Dorcadion}

Term. Füzetek 15, 133

Mehrere Syntypen, Hungaria, durch CERVA.

SDEI: 3 Syntypen, Hungaria, Coll. KLÄGER.

\section{chloe GounelLe, 1911, Callichroma}

Bull. Soc. ent. Fr. 1911, 167-168

11 Syntypen, Brasilien, Bahia, Minas, Sao Paulo, Sta Catharina, im Museum Paris, Coll. WAgner, im SDEI und in Coll. Gounelle.

SDEI: 4 Syntypen, Brasilien, Bahia, leg. Fruhstorfer; Sao Paulo, leg. Metz, Coll. KraAtz.

christophi KRaAtz, 1879, Clytus

Dtsch. ent. Ztschr. 23, 108-109, Taf. 1, Fig. 4

Mehrere Syntypen, Ost-Sibirien, Amur, leg. Christoph. SDEI: 8 Syntypen, Amur, 1877, leg. Christoph, Coll. KraAtz und Coll. v. Heyden.

chrysomaculata Schwarzer, 1925, Glenea

Ent. Bl. 21, 152-153

Mehrere Syntypen, Formosa: Kosempo, 7.VII.1909/ VI.1911/ 22.VI.1912; Sokutsu, 22.VI.1912; Alikang, 7.VIII.; Kankau, IV., V., 7.VI.1912; leg. SAUter.

SDEI: Formosa, leg. SAUTER, Syntypen: 7 Ex. Kosempo, VI.1911/ 22.V., 22.VI.1912; 1 Ex. Alikang, 7.VIII.; 1 Ex. Sokutsu, Banshoryo Distr., 22.VI.1912; 13 Ex. Kankau, Koshun, 7.IV., IV., V., VI.1912 (als var. conjuncta SCHWARZER bezettelt).

cinerascens KraAtz, 1882, Phytoecia

Dtsch. ent. Ztschr. 26, 337-338

Mehrere Syntypen, Samarkand, leg. Haberhauer. SDEI: 1 Syntypus, Samarkand, 1881, durch STAUdINGER, Coll. KraAtz.

\section{cineriferum Suv0Rov, 1909, Dorcadion}

Rev. Russe d'Ent. 9, 290-291

$20^{\star}, 2$ ㅇ Syntypen, Transkaukasien, Umgebung des Goktsha Sees, 7.V.1909, leg. Maljuzhenko.

SDEI: 1 o Syntypus, Caucasus, Goktsha, leg. MaljuzHENKo, Coll. KLÄGER.

\section{cingulatum Ganglbauer, 1884, Dorcadion}

Verh. Zool. Bot. Ges. Wien 1883, 484-485

Mehrere Syntypen, Persien, durch Kraatz und Plason. SDEI: 6 Syntypen, ohne Fundort, Coll. KraAtz.

circumcinctum AurIVILLIUS, 1911, Obrium

Arch. för Zool. 7 (1), 5 (1910)
Mehrere Syntypen, Deutsch-Ost-Afrika, Usambara, im SDEI und Museum Stockholm.

SDEI: 8 Syntypen, Ostafrika, Usambara, Coll. KraATz.

circumdata KraAtz, 1882, Phytoecia

Dtsch. ent. Ztschr. 26, 337

Mehrere Syntypen, Samarkand, leg. Haberhauer.

SDEI: 2 Syntypen, Samarkand, 1881, durch STAUDINGER, Coll. Kratiz.

\section{circumscriptus Schwarzen, 1925, Chlorophorus}

Ent. Bl. 21, 28

Mehrere Syntypen, Formosa: Kosempo, 7.IX.1909/ 7.IX.1911; Fuhosho, 7.VII.; Taihorin, 22.VI.; Sokutsu, 22.VI.1912; leg. SAUTER.

SDEI: Formosa, leg. SAUter, Syntypen: 3 Ex. Kosempo, 7.IX.1909/1911; 1 Ex. Fuhosho, 7.VIII. (nicht 7.VII.!); 1 Ex. Sokutsu, 22.VI.1912.

\section{colchica ReITTER, 1890, Cortodera}

Wien. ent. Ztg. 9, 246

Mehrere Syntypen, Kaukasus, Araxesthal.

SDEI: 3 Syntypen, Caucasus, Araxesthal, leg. LederReitter, Coll. Stierlin, Coll. F. Müller, Coll. v. Heyden.

complanatum GANGlBaUeR, 1884, Dorcadion

Verh. Zool. Bot. Ges. Wien 1883, 484

Mehrere Syntypen, Persien, durch Plason.

SDEI: 1 Syntypus, Persien, Coll. KraAtz.

concentricale JoRDAN, 1894, Callichroma

Novit. Zool. 1, 166

Mehrere Syntypen, Afrika; Kuila, 1892, leg. Mocquerys; Loanda; im Tring Museum.

SDEI: 1 Syntypus, Loanda, Coll. KraAtz.

congolensis HINTZ, 1911, Velleda

Wiss. Erg. Zentr. Afr. Exp. (1907/08), Zool. 3, 434

7 Syntypen, Congo Belge, Kondué, leg. LujA.

SDEI: 3 Syntypen, mit den Angaben der Beschreibung.

conradti AuRIVILlius, 1907, Apiogaster

Dtsch. ent. Ztschr. 1907, 78

Mehrere Syntypen, Kamerun, leg. ConradT, via SCHenKLING an AURIVILLIUS, im SDEI.

SDEI: 1 Syntypus, Kamerun, leg. Conradt, Coll. KraAtZ.

conradti KolBe, 1893, Ceroplesis

Stett. ent. Ztg. 54, 265-266

Mehrere Syntypen, Deutsch-Ostafrika, Usambara, Derema, E.VIII.-XII.1891, leg. ConRADT.

SDEI: 1 Syntypus, Usambara, Coll. KraATZ.

conradti AuRIVILLIUS, 1907, Leiopus

Dtsch. ent. Ztschr. 1907, 82-83

Mehrere Syntypen, Kamerun, leg. ConRadT, im SDEI. SDEI: 10 Syntypen, Kamerun, leg. Conradt, Coll. KraAtz. 
conradti AuRIVILLIUS, 1907, Sophronica

Dtsch. ent. Ztschr. 1907, 81

Mehrere Syntypen, Kamerun, leg. ConRadT, im SDEI.

SDEI: 1 Syntypus, Kamerun, leg. Conradt, Coll. KraAtZ.

convexa Broun, 1893, Somatidia

Man. N. Zeal. Col. 7, 1498

Mehrere Syntypen, New Zealand, Maketu, Hunua Range.

SDEI: 2 Syntypen, Auckland, Maketu, Hunua, Coll. Broun.

cribricolle KraAtZ, 1882, Dorcadion

Dtsch. ent. Ztschr. 26, 196

Mehrere Syntypen, Altai, in Coll. Krattz und Coll. STAUDINGER.

SDEI: 1 Syntypus, Altai, durch Staudinger, Coll. KRAATZ.

\section{cribrithorax KoLBE, 1897, Plocederus}

Käf. Dtsch. Ost Afr., 297

Mehrere Syntypen, Dar-es-Salaam, VIII.1894, leg. STUHLMANN und leg. v. BENNigSEN.

SDEI: 4 Syntypen, Dar-es-Salaam, leg. v. Bennigsen, Coll. v. Bennigsen.

cuneipennis KraAtz, 1879, Clytus

Dtsch. ent. Ztschr. 23, 110-111, Taf. 1, Fig. 5

Holotypus, Ost-Sibirien, Amur, leg. Christoph, im SDEI, Coll. KraATZ, 1877 (als cuneiformis bezettelt!).

curticornis GanglBauen, 1888, Prionus

Dtsch. ent. Ztschr. 1888, 266-268

$\sigma^{\top}$ Holotypus, Turkestan, Farab., leg. Russow, in Coll. v. Heyden, im SDEI.

curvipes HINTZ, 1911, Nosoeme

Wiss. Erg. Zentr. Afr. Exp. (1907/08) Zool. 3, 426

1 Ex. Beni (Urwald), w.v. Ruwenzori, II.1908; 6 Ex.

Kondué, Congo-Belge, leg. Luja: Syntypen.

SDEI: 2 Syntypen, Congo-Belge, Kondué, leg. LuJA.

\section{debilis GounelLe, 1911, Charisia}

Ann. Soc. ent. Fr. 80, 59-60

$100^{\star}, 9$ ㅇ Syntypen, Brasilien, Prov. Goyaz, Jatahy.

SDEI: 2 Syntypen, mit den Angaben der Beschreibung,

Coll. KraAtz.

debilis KraAtz, 1879, Grammoptera

Dtsch. ent. Ztschr. 23, 104-105

Holotypus, Ost-Sibirien, Amur, leg. Christoph, im SDEI, Coll. KraATZ, 1877.

\section{decolorata SchWARzER, 1925, Cereopsius}

Ent. Bl. 21, 59

Mehrere Ex. Kosempo, VI., XII.1909/ 7.X.1911/ 22.VI. 1912; Sokutsu, 7.VIII.1912; 2 우, Pilam, VII.1912: Formosa, leg. SAUTER, Syntypen.
SDEI: Formosa, leg. SAUTER, Syntypen: 8 Ex. Banshoryo Distr., Sokutsu, 7.VII., 7.VIII.1912; 2 Ex. Kosempo, 22.V., 22.VI.1912; 1 Ex. Pilam, VIII.1912 (als var. annulicornis SCHWARZER bezettelt).

decorata Nonfried, 1894, Clodia

Dtsch. ent. Ztschr. 1894, 214-215

Mehrere Syntypen, N. O. Sumatra, Tebing-Tinggi, leg. Schultheiss, in Coll. Schultheiss.

SDEI: 2 Syntypen, N. O. Sumatra, Tebing-Tinggi, leg. SChULtheiss.

detrita KraAtz, 1882, Agapanthia

Dtsch. ent. Ztschr. 26, 336

Mehrere Syntypen, Samarkand, leg. Haberhauer.

SDEI: 1 Syntypus, Samarkand, durch STAUDINGER, 1881, Coll. KraAtZ.

deyrollei GanglBaUeR, 1884, Dorcadion

Verh. Zool. Bot. Ges. Wien 33 (1883), 482-483

2 o Syntypen, Transcaucasien, Bitlis, leg. Deyrolle, durch KRAATZ und OBERThür.

SDEI: 1 Syntypus, ohne Fundort, leg. Deyrolle, Coll. KraAtz.

discicollis HeYDEN, 1876, Molorchus

Dtsch. ent. Ztschr. 20, 383-384

o Holotypus, Eichberg im Rheingau, leg. Richter, in Coll. v. Heyden, im SDEI.

discolor GOUNELLE, 1911, Ommata

Ann. Soc. ent. Fr. 80, 34-35

$14 \sigma^{\pi}, 22$ ? Syntypen, Brasilien, Prov. Goyaz, Jatahy.

SDEI: 4 Syntypen, mit den Angaben der Beschreibung, Coll. KraAtz.

\section{distinguendum GounelLe, 1911, Callichroma}

Bull. Soc. ent. Fr. 1911, 165-167

5 Syntypen, Brasilien, Minas Geraes, Sao Paulo, im SDEI und in Coll. Gounelle.

SDEI: 1 Syntypus, Brésil, Coll. KraAtz.

\section{diversenotata SCHWARzER, 1925, Glenea}

Ent. Bl. 21, 152

Holotypus, Formosa, Kosempo, 7.VI.1912, leg. SAUTER, im SDEI.

\section{dsungaricum PIC, 1907, Dorcadion}

Echange 23, 104 (Beschreibung), weitere Angaben In: Mat. Longicorn. 6 (2), 11; 1907

Mehrere Syntypen, Dsungaria, Borochoro, Coll. Hauser und Coll. Pic.

SDEI: 12 Syntypen, Dsungaria, Borochoro-Geb., VI.1905, durch Leonhard, Coll. Hauser (6 Ex. als var. melancholium Pic bezettelt). 
duplicatum GounelLe, 1909, Ibidion

Ann. Soc. ent. Fr. 77, 677

12 Syntypen, Brasilien, Prov. Goyaz, Jatahy.

SDEI: 1 Syntypus, Brésil, Prov. Goyaz, Jatahy, Coll.

KRAATZ.

elegans KraATZ, 1873, Dorcadion

In: Küster, Käf. Europ. 29, 73

Mehrere Syntypen, bei Sarepta, leg. Becker und leg. CHRISTOPH.

SDEI: 7 Syntypen, Sarepta, leg. Becker und leg. ChrisTOPH, Coll. KRAATZ.

elegans GouneLLE, 1909, Ibidion

Ann. Soc. ent. Fr. 77, 679

5 Syntypen, Brasilien, Prov. Goyaz, Jatahy.

SDEI: 1 Syntypus, Brésil, Prov. Goyaz, Jatahy, Coll. KRAATZ.

elegans VILLIERS, 1980, Nethinius

Faun. Madagasc. 52, 43, 101, Fig. 273-275

$0^{\top}$ Holotypus, Madagascar, Ambodivoangy, XII.1945, leg. VAdon, im Museum Paris, Coll. VAdon; mehrere Paratypen, siehe Originalbeschreibung.

SDEI: 1 Paratypus, Madagaskar, Coll. KraATZ.

elegans Nonfried, 1894, Pithomictus

Dtsch. ent. Ztschr. 1894, 213-214

Mehrere Syntypen, N. O. Sumatra, Tebing-Tinggi, leg. Schultheiss, in Coll. Schultheiss.

SDEI: 2 Syntypen, mit den Angaben der Beschreibung, 22.VI.1884/ 11.II.1885.

elegantula KraATz, 1879, Grammoptera

Dtsch. ent. Ztschr. 23, 105-106

$10^{\top}, 1$ ㅇ Syntypen, Ost-Sibirien, Amur, leg. Christoph, im SDEI, Coll. KraATZ.

\section{erythrospila HeLLER, 1912, Trigonoptera}

Ent. Mitt. 1, 172-173, Fig. 3

Mehrere Syntypen, Nova Guinea Germanica, Coll. v. BeNNigSEN.

SDEI: 2 Syntypen, D. N. Guinea, leg. und Coll. v. BENNIGSEN.

excellens AuriviluIus, 1908, Tmesisternus

Dtsch. ent. Ztschr. 1908, 214, Taf. 3, Fig. 5

Mehrere Syntypen, Deutsch Neu-Guinea, Sattelberg, leg. v. BENNIGSEN.

SDEI: 1 Syntypus, mit den Angaben der Beschreibung.

excellens inflatus Quentin \& VILLIERS, 1972, Pixodarus Ann. Soc. ent. Fr. 8 (N. S.), 288

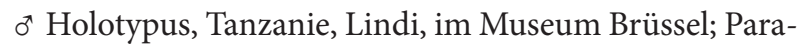
typen, siehe Originalbeschreibung.

SDEI: 1 o Paratypus, Dar-es-Salaam, leg. v. BenNigsen, Coll. v. Bennigsen. excellens konduanum ScHMIDT, 1922, Metallichroma

Arch. Naturg. 88, A 6, 132

Holotypus, Belg. Kongo, Kundué, leg. LuJA, im SDEI.

excelsa StierLin, 1876, Phytoecia

Mitt. Schweiz. ent. Ges. 4 (1874), 479

Mehrere Syntypen, ohne Fundort.

SDEI: Lectotypus, Sarepta, Coll. StiERLin, Designation: SAMA.

exigua AurivilLius, 1907, Eunidia

Dtsch. ent. Ztschr. 1907, 80

Mehrere Syntypen, Kamerun, leg. ConRadt, im SDEI.

SDEI: 1 Syntypus, Kamerun, leg. ConradT, Coll. KraAtZ.

exigua AuriviLiuUs, 1907, Sophronica

Dtsch. ent. Ztschr. 1907, 81

Mehrere Syntypen, Kamerun, leg. ConRadT, im SDEI.

SDEI: 1 Syntypus, Kamerun, leg. Conradt, Coll. KraAtz.

fairmairei GounelLe, 1909, Ibidion

Ann. Soc. ent. Fr. 77, 680

Mehrere Syntypen, Brasilien, Prov. Goyaz, Jatahy.

SDEI: 1 Syntypus, Brésil, Prov. Goyaz, Jatahy, Coll. KraAtz.

faldermanni GanglBaUeR, 1884, Dorcadion

Verh. Zool. Bot. Ges. Wien 33 (1883), 493

Holotypus, Persien, durch KraAtz, im SDEI.

fallax HeLlen, 1935, Demonax

Arb. morph. tax. Ent. 2, 267, Fig. 3

Mehrere Syntypen, Salomo-Inseln, leg. Malcher, im Museum Dresden und SDEI.

SDEI: 1 Syntypus, Salomo I., Neu Georgio, Pauru, leg. MALChER.

fallax KraAtz, 1873, Dorcadion

In: Küster, Käf. Europ. 29, 89

Mehrere Syntypen, Rumelien, bei Saloniki, leg. RayMOND, Türkei, durch v. Frivaldszky, im Museum Berlin und in Coll. KraAtz.

SDEI: Lectotypus, Saloniki, Coll. KraAtz, Designation: SAMA.

\section{fasciata AurivilLius, 1907, Nupserha}

Dtsch. ent. Ztschr. 1907, 83-84

Mehrere Syntypen, Togo, leg. ConRadT, im SDEI.

SDEI: 1 Syntypus, Togo, leg. Conradt, Coll. KraATz.

\section{fasciata AuriviLLIUS, 1908, Ocularia}

Dtsch. ent. Ztschr. 1908, 79

Mehrere Syntypen, Kamerun, leg. ConRadT, via SchenkLING an AURIVILLIUS, im SDEI.

SDEI: 1 Syntypus, Kamerun, leg. Conradt, Coll. KraAtz. 
fasciata SchWARzer, 1925, Pseudale

Ent. Bl. 21, 67

Holotypus, Formosa, Fuhosho, 7.VIII., leg. SAUter, im SDEI.

femoralis AuRIVILLIUS, 1907, Eunidia

Dtsch. ent. Ztschr. 1907, 80

Mehrere Syntypen, Kamerun, leg. ConRadT, im SDEI.

SDEI: 1 Syntypus, Kamerun, leg. ConradT, Coll. KRAATZ.

ferranti HINz, 1913, Isosaphanus

Dtsch. ent. Ztschr. 1913, 196

Mehrere Syntypen, Congo Belge, Kondué.

SDEI: 1 Syntypus, Congo Belge, Kondué, leg. LujA, Coll. KRAATZ.

ferrugator namaensis FIEDLER, 1938, Ceroplesis

Dtsch. ent. Ztschr. 1938, 589

Holotypus, 19 Paratypen, Afrika, Groß-Namaland, Namaqualand, Kubub; im Museum Berlin, Senckenberg Museum und SDEI.

SDEI: 2 Paratypen, DSWA, Kubub, Coll. v. Bennigsen.

ferrugineus KraAtz, 1863, Cyamophthalmus

Berl. ent. Ztschr. 7, 100, Taf. 4, Fig. 6a

Holotypus, Andalusien, via Staudinger an KraAtz, im SDEI.

\section{flavovittatus Aurivillius, 1908, Paratrypanius}

Dtsch. ent. Ztschr. 1908, 223-224, Fig. 12

Mehrere Syntypen, Samoa, Apia, leg. v. Bennigsen.

SDEI: 1 Syntypus, Apia, Coll. v. BenNigsen.

\section{forcipiferum KRAATZ, 1873, Dorcadion}

In: Küster, Käf. Europ. 29, 60

Mehrere Syntypen, Palästina, leg. Roтн.

SDEI: 1 Syntypus, Palästina, leg. Roth, Coll. KraATZ.

formosana SCHWARzer, 1925, Agelasta

Ent. Bl. 21, 61

Mehrere Syntypen, Formosa: Fuhosho, VI.1909/ 7.IX. 1910; Kosempo, VI.1909; leg. SAuter.

SDEI: Formosa, leg. SAUter, Syntypen: 2 Ex. Fuhosho, 7.IX., VI.1909; 1 Ex. Kosempo, VI.1909.

\section{formosana SCHWARZER, 1925, Daphisia}

Ent. Bl. 21, 153

Mehrere Syntypen, Formosa: Taihorinsho, 7.XI.; Taihorin, 7.VI.1911/ 22.VI.; Kosempo, 7.VII.1911; Pilam, VII.1912; leg. SAUTER.

SDEI: Formosa, leg. SAuter, Syntypen: 3 Ex. Taihorin, 7.VI.1911/ 22.VI.; 2 Ex. Taihorinsho, 7.IX. (nicht 7.XI!); 1 Ex. Kosempo, 7.VII.1911; 1 Ex. Pilam, VI.1912 (nicht VII.!).
Mehrere Syntypen, Formosa, Kankau, V., VI.1912, leg. SAUTER.

SDEI: 24 Syntypen, Formosa, Kankau, Koshun, 7.IV., 7.V., V., VI., VII.1912, leg. Sauter (16 Ex. als ab. lineatomaculata SCHWARZER bezettelt).

\section{formosana SchWARzer, 1925, Mesosa}

Ent. Bl. 21, 60-61

Mehrere Syntypen, Formosa, Hoozan, 7.IX.1910, leg. SAUTER.

SDEI: 2 Syntypen, mit den Angaben der Beschreibung.

formosana Schwarzer, 1929, Pothyne

Ent. Bl. 21, 146

Mehrere Syntypen, Formosa: Kosempo, 7.VII.1911; Kankau und Sokutsu, 7.VII.1912; leg. SAUTER.

SDEI: Formosa, leg. SAuter, Syntypen: 1 Ex. Kosempo, 22.VI.1911; 1 Ex. Banshoryo-Distr., Sokutsu, 7.VII.1912.

\section{formosana SCHWARzER, 1925, Trirachys}

Ent. Bl. 21, 21

Mehrere Syntypen, Formosa: Kankau, V.1912; Kosempo, VII.1911/ VII.1912; leg. SAUTER.

SDEI: Formosa, leg. SAUter, Syntypen: 2 Ex. Kosempo, 22.V., VII.1911; 1 Ex. Kankau, Koshun, V.1912.

\section{formosanum SCHWARZER, 1925, Obrium}

Ent. Bl. 21, 22-23

Mehrere Syntypen, Formosa, Kosempo, V., 7.VI.1912, leg. SAUTER.

SDEI: 2 Syntypen, Formosa, Kosempo, V., 7.VI.1912, leg. SAUTER.

\section{formosanus SchWarzer, 1925, Pyrestes}

Ent. Bl. 21, 24

Mehrere Syntypen, Formosa: Fuhosho, V.1909; Kosempo, 22.V.1912; Sokutsu, 7.VII.1912; leg. SAUTER.

SDEI: Formosa, leg. SAUter, Syntypen: 5 Ex. Kosempo, 22.V., VI.1912; 2 Ex. Banshoryo-Distr., Sokutsu, 7.VI., VII.1912; 3 Ex. Fuhosho, V.1909.

\section{formosanus SCHWARzer, 1925, Thranius}

Ent. Bl. 21, 23

Mehrere Syntypen, Formosa: Fuhosho, VI.1909; Hoozan, 7.IX.; Kosempo, 7.IX.1911/ 7.X.1911/ 22.V.1912; leg. SAUTER.

SDEI: Formosa, leg. SAuter, Syntypen: 2 Ex. Kosempo, 7.IX.1911/ 22.V.1912; 1 Ex. Fuhosho, VI.1909; 1 Ex. Hoozan, VIII.1910.

\section{formosanus SCHWARZER, 1925, Xylotrechus}

Ent. Bl. 21, 26

Mehrere Syntypen, Formosa, Alikang, 7.X., leg. SAUTER.

SDEI: 4 Syntypen, Formosa, Alikang, 7.X., leg. SAUTER.

formosana SCHWARzER, 1925, Glenea

Ent. Bl. 21, 150 
formosum Suvorov, 1910, Compsodorcadion

Rev. Russe d'Ent. 10, 65-66

5 o, 1 \& Syntypen, Semirjetshje Gebiet, i. d. Schlucht Kop-muly des Fl. Usun-Kargaly, 15.IV.1909, leg. NiedZWIECKI

SDEI: $1 \sigma^{*}$ Syntypus, Semirjetshje, Kop-mula, Coll. KL $\ddot{A}-$ GER.

\section{formosum Kratz, 1870, Dorcadion}

Berl. ent. Ztschr. 14, 411-412, Taf. 3, Fig. 4a

Mehrere Syntypen, Kaukasus, via Manke an KraAtz.

SDEI: 1 Syntypus, Caucausus, 5.VII.1870, Coll. KraATz.

\section{frivaldszkyi KraATz, 1876, Grammoptera}

Dtsch. ent. Ztschr. 20, 344

Mehrere Syntypen, bei Budapest.

SDEI: Lectotypus, 1 Paralectotypus, Ungarn, in Coll. v. Heyden; 3 Paralectotypen, Pest, Designation: Sama.

\section{fugitivus THIEME, 1881, Clytus}

Berl. ent. Ztschr. 25, 100-101

7 Syntypen, Amur, via Staudinger an Thieme.

SDEI: 2 Syntypen, Amur, leg. Christoph, 1871.

\section{fulgidipennis GounelLe, 1909, Haruspex}

Ann. Soc. ent. Fr. 77, 652-653

9 Syntypen, Brasilien, Prov. Goyaz, Jatahy.

SDEI: 1 Syntypus, Brésil, Prov. Goyaz, Jatahy, Coll. KRAATZ.

\section{fulva SCHWARZER, 1925, Atimura}

\section{Ent. Bl. 21, 145}

Mehrere Syntypen, Formosa, Kankau, VI.1912, leg. SAUTER.

SDEI: 1 Syntypus, Formosa, Kankau, Koshun, XI.1912 (nicht VI!), leg. SAuter.

\section{fulvonotata Nonfried, 1894, Merionoeda}

Dtsch. ent. Ztschr. 1894, 207-208

Mehrere Syntypen, N. O. Sumatra, Tebing-Tinggi, leg. SCHUltheiss.

SDEI: 8 Syntypen, mit den Angaben der Beschreibung, 29.II., 7.IX., 8.IX.1884, Coll. Schultheiss.

\section{funestum GANgLBAUER, 1884, Dorcadion}

Verh. Zool. Bot. Ges. Wien 33 (1883), 501

Mehrere Syntypen, Mytilene.

SDEI: 2 Syntypen, Mytilene, Coll. KraAtz.

\section{fuscicornis Heyden, 1863, Phytoecia}

Berl. ent. Ztschr. 7, 130

Holotypus, Algier, im SDEI, Feisth., Riese, Coll. v. HEYDEN.

\section{fuscus NonfRied, 1894, Trypogeus}

Dtsch. ent. Ztschr. 1894, 209-210

Mehrere Syntypen, N. O. Sumatra, Tebing-Tinggi, leg. Schultheiss, in Coll. Schultheiss.
SDEI: 1 Syntypus, mit den Angaben der Beschreibung, 12.V.1884.

\section{ganglbaueri KraAtz, 1884, Mallosia}

Dtsch. ent. Ztschr. 28, 233-234

Mehrere Syntypen, südl. Kleinasien, Malatia.

SDEI: Lectotypus, Malatia, Coll. KraAtz, Designation: SAMA.

\section{ganglbaueri ReITTER, 1894, Morimus}

Wien. ent. Ztg. 13, 44

Mehrere Syntypen, Bosnien.

SDEI: 1 Syntypus, Bosnien, durch Leder-Reitter, Coll. v. Heyden.

\section{gebleri KraAtz, 1873, Dorcadion}

In: Küster, Käf. Europ. 29, 33

Mehrere Syntypen, in den Steppen der Wolga.

SDEI: 1 Syntypus, ohne Fundort, Coll. KraATz.

\section{genisspina SCHWARZER, 1923, Steirastoma}

Dtsch. ent. Ztschr. 1923, 259-260

3 ơ, 1 ㅇ Syntypen, Corumba, Matto Grosso, via StaUDINGER an SCHWARZER.

SDEI: 1 Syntypus, Corumba, Matto Grosso.

\section{glabrofasciatum DANIEL, 1901, Dorcadion}

Soc. Ent. 15, 140

Mehrere Syntypen, Asia minor, leg. Escherich und leg. v. BODEMEYER.

SDEI: 24 Syntypen, Asia Minor, Biledjik, Eski Chehir, leg. v. Bodemeyer, Coll. Leonhard, Coll. KLÄger, Coll. v. Heyden (3 Ex. als var. imparivittatum DANIEL bezettelt).

glazunovi Suvorov, 1910, Compsodorcadion

Rev. Russe d'Ent. 10, 69-70

Mehrere Syntypen, Semirjetschje-Gebiet, im Bezirk Dsharkent, am Fluß Ili, IV., V.

SDEI: 2 Syntypen, Dzhungaria ross, Dsharkent, am Fluß Ili, 14.IV.1906, leg. RüCKBEIL, Coll. KLÄGER.

\section{globithorax radkevitshi Suvorov, 1910 Compsodorcadion}

Rev. Russe d'Ent. 10, 61-62

Mehrere Syntypen, Ala-tau, Paßhöhe Kurdaisk, Mitte V.1907, leg. Jacobson, Coll. Semenov-Tian-Shansky. SDEI: 3 Syntypen, Semiretschije Kurdai, 14.V.1907, leg. Jacobson, Coll. KLÄger, Coll. v. Heyden.

\section{gracilis KraAtz, 1873, Xylosteus}

Berl. ent. Ztschr. 17, 202

$10^{*}$ Syntypus, Illyrien; $10^{*}$ Syntypus, Griechenland; $10^{*}$ Syntypus „ein nicht recht ausgefärbtes caucasisches Ex.", ex Coll. Lederer, in Coll. TürK. SDEI: 1 Syntypus, „Karst“. 
graecum KRAATZ, 1873, Dorcadion

In: Küster, Käf. Europ. 29, 78; nom praeocc. = olympicum Ganglbauer, 1882, Wien. ent. Ztg. 1, 228

Mehrere Syntypen, Brussa, Olymp, durch v. Frivaldszky; Constantinopel, leg. PrÖMmel; Griechenland, leg. ZeBE. SDEI: 17 Syntypen, Asia minor, Brussa, Graecia, leg. Zebe, in Coll. KraAtz, Coll. KLÄGer, (2 Ex. als var. subalpinum KraAtz und 7 Ex. als ab. obsoletum KraATz bezettelt).

\section{grandis SCHWARzER, 1929, Apomecyna}

Senckenbergiana 11, 375-376, Fig. 32

ㅇ Holotypus, 1 ㅇ Paratypus, D.-O.-Afrika, Amani, im SDEI und Senckenberg Museum Frankfurt a. M.

SDEI: Holotypus, Amani, leg. ZimmermanN.

\section{granulosus ScHWARzer, 1924, Anancylus}

Treubia 5, 237

Mehrere Syntypen, Süd-Sumatra, Wai Lima, Lampongs, XI.-XII.1921, leg. KarnY \& Siebers.

SDEI: 1 Syntypus, mit den Angaben der Beschreibung, ex Museum Buitenzorg.

\section{griseopennis SCHWARZER, 1925, Oberea}

Ent. Bl. 21, 154

Mehrere Syntypen, Formosa: Kankau, 7.IV.1912; Kosempo, 22.V.1912; Sokutsu, 7.VI.1912; leg. SAUTER.

SDEI: Formosa, leg. SAUter, Syntypen: 3 Ex. Kosempo, 22.V.1912/ 22.VII.; 1 Ex. Kankau, Koshun, V.1912; 1 Ex. Sokutsu, Banshoryo Distr., 7.VI.1912.

\section{grumi Suvorov, 1909, Neodorcadion}

Rev. Russe d'Ent. 9, 80-82

66 o , 5 i Syntypen, nordwestl. Mongolei, am Fluß Namura, nördlich von Kobdo, Mitte VII.1903 und nördlich des Gebirgsrückens Tanny-Ola, Anfang VII.1903, Exped. von Grum-Grzhimailo, Coll. Semenov-TianSHANSKY.

SDEI: 1 Syntypus, Kobdo, Fluß Namur, 23.VII.1903, leg. Grum, Coll. KLÄGER.

\section{guldei Chоваuт, 1905, Tetropiopsis}

Bull. Soc. ent. France 1905, 157

○ Holotypus, Algérie méridionale.

SDEI: Holotypus, El Kantara, 7.VII.1904, leg. GuLDE.

\section{halepense KraATZ, 1873, Dorcadion}

In: Küster, Käf. Europ. 29, 72; als var. von saulcyi ThомsoN beschrieben

Mehrere Syntypen, Aleppo.

SDEI: 1 Syntypus, Aleppo, Coll. KraAtz (als aleppense KRAATz bezettelt).

\section{hamatus AuriviLLIUs, 1907, Timoreticus}

Dtsch. ent. Ztschr. 1907, 79

Mehrere Syntypen, Westafrika, Bojuma, via ScHENKLING an Aurivillius, im SDEI.

SDEI: 1 Syntypus, W. Afrika, Bojuma, Coll. KraAtz. hauseri ReITTER, 1895, Dorcadion

Wien. ent. Ztg. 14, 160-161

Mehrere Syntypen, Thian-Schan, im südöstlichen Turkestan, leg. Hauser.

SDEI: 4 Syntypen, Thian-Schan, Musart, VI.1894, Coll. HAUSER, durch LEONHARD.

\section{heldreichi KRAATz, 1873, Dorcadion}

In: KüsteR, Käf. Europ. 29, 90

Mehrere Syntypen, auf dem Parnass bei Athen, leg. v. Heldreich, Coll. v. Kiesenwetter.

SDEI: 2 Syntypen, Parnass, Coll. KraAtz.

hellmanni GanglBaueR, 1884, Dorcadion

Verh. Zool. Bot. Ges. Wien 33 (1883), 486

Mehrere Syntypen, Persien, durch v. Frivaldszky, KraAtz und Plason.

SDEI: 6 Syntypen, Persien, leg. Plason, Coll. KraAtz.

herminae ReITTER, 1890, Mallosia

Wien. ent. Ztg. 9, 241

Mehrere Syntypen, Araxesthal.

SDEI: 10 Syntypen, Caucasus, Araxesthal, leg. LederReitter, Coll. KraAtz, Coll. Franklin Müller, Coll. KraAtz, Coll. v. Heyden.

\section{heteroclita GouneLLe, 1909, Cupanoscelis}

Ann. Soc. ent. Fr. 77, 619, Fig. 15

Mehrere Syntypen, Brasilien, Prov. Goyaz, Jatahy, Umgebung von Cidade de Condeuba, Bahia, Sertão de Diamantina, Minas.

SDEI: 1 Syntypus, Brésil, Prov. Goyaz, Jatahy, Coll. KraAtZ.

\section{heurni Schwarzer, 1924, Arrhenotus}

Nova Guinea 15, 58-59, Fig. 11, 11a

Mehrere Syntypen, N. West Neu-Guinea, Prauwenbivak, Idenburg River, XI.-XII.1920, leg. v. HeurN, Holländ. Exped.

SDEI: 1 Syntypus, mit den Angaben der Beschreibung, ex Museum Buitenzorg.

heydeni StierLin, 1864, Clytus

Berl. ent. Ztschr. 8, 152-153

$1 \sigma^{x}, 1$ i Syntypen, Sicilien, im SDEI, Coll. StIERLIN.

heydeni KraAtz, 1870, Dorcadion

Berl. ent. Ztschr. 14 (Beiheft), 161-163

1 ơ $^{\star}, 1$ q Syntypen, Albas, leg. v. Heyden, via v. Heyden an KraATZ.

SDEI: Lectotypus, 1 Paralectotypus, Albas, Coll. KraAtz, Coll. v. Heyden, Designation: Vives, 1979.

\section{heydeni BaECKMAnN, 1923, Hesperophanes}

Rev. Russe d'Ent. 18, 67-68, Fig.

1 ㅇ, Stadt Perovsk am Syr-Darja, 15.VII.1905, leg. SCHEll; 1 + , Dzbulek, südl. von Perovsk, 9.VII.; 2 o, dito, 28.VII.1911; leg. Koshantshikov; $10^{\star}$, dito, 25.VI.1915, 
leg. Pavlovskij; im Zoologischen Museum der Akademie der Wissenschaften Leningrad und in Coll. BAECKMANN: Syntypen.

SDEI: Paralectotypus, Syr-Daria, Baigacum, 28.VII.1911, leg. Kozhantschikoff, Designation: Sama.

heydeni Ganglbauer, 1888, Phytoecia

Dtsch. ent. Ztschr. 32, 76-77

Holotypus, Mesopotamien, Malatia, im SDEI 1885 durch Staudinger, Coll. v. Heyden.

heydeni Ganglbauer, 1888, Prionus

Dtsch. ent. Ztschr. 32, 265-266

o Holotypus, Turkestan, Namangan, in Coll. v. Heyden, im SDEI 1885 durch STAUdINGER.

hilairei GOUNELLE, 1909, Mallocera

Ann. Soc. ent. Fr. 77, 648-649

Mehrere Ex. Brasilien, Prov. Goyaz, Jatahy; 1 Ex. Serra de Communaty, Pernambuco; Syntypen.

SDEI: 1 Syntypus, Brésil, Prov. Goyaz, Jatahy, Coll. KrAATZ.

holubi bifoliata SCHMIDT, 1922, Phyllocnema

Arch. Naturg. 88, A 6, 177

Mehrere Syntypen; Afrika, Mpapua, I.1899, leg. KAnnenBERG, im Museum Berlin; Uhehe, Iringa, I.-III.1899, leg. GöTzE, im Museum Berlin; Usaramo, im Museum Berlin und SDEI; Tanganjika, Kasanga, leg. Speyer, im Museum Hamburg.

SDEI: 1 Syntypus, Usaramo, Coll. v. Bennigsen.

\section{horni HeLlen, 1912, Megaceresium}

Ent. Mitt. 1, 171, Fig. 1

Mehrere Syntypen, Salomo Insel und Nova-Pommerania, penninsula Gazelle, Coll. v. Bennigsen.

SDEI: 2 Syntypen, H. Insel Gazelle, D. N. Guinea, Salomons Insel, Coll. v. BenNigsen.

hornianum PLAVILSTSHIKOV, 1958, Dorcadion

Fauna USSR n.F. 70, 23, 394-396

$\sigma^{\star}$ Holotypus, $1 \sigma^{\star}, 1$ ㅇ Paratypen, Chinesische Dsungarei, Borochoro Gebirge, VI.1905, im SDEI und in Coll. Plavilstshikov.

SDEI: ơ Holotypus, 1 o Paratypus, mit den Angaben der Beschreibung, leg. Leonhard, Coll. Hauser.

\section{hottentotta transvaalensis FIEDLER, 1938, Ceroplesis}

Dtsch. ent. Ztschr. 1938, 612

Holotypus, 11 Paratypen, Afrika, Transvaal, Lydenburg, Mpome, im Museum Berlin, Museum Brüssel, Senckenberg Museum, Frankfurt a. M. und SDEI.

SDEI: 1 Paratypus, Transvaal, leg. Hartmann, Coll. KRAATZ.

hybridula ReITTER, 1901, Strangalia

Dtsch. ent. Ztschr. 1901, 188 $20^{\star}$, Spanien, Albas, Asturien; $1 \sigma^{\star}, 1$ ㅇ, Portugal, Sierra do Gerez, in Coll. v. Heyden; Syntypen.

SDEI: Syntypen, Portugal: 1 Ex. Albas; 2 Ex. Sr. Gerez, Coll. v. Heyden.

\section{illyricus KraAtz, 1870, Xylosteus}

Berl. ent. Ztschr. 14, 415

o Syntypen, Illyrien.

SDEI: đ Lectotypus, „Karst“, Designation: SAma (Biocosme Mésogéen 10, 13; 1993).

impressicolle KraATz, 1873, Dorcadion

In: Küster, Käf. Europ. 29, 47

Mehrere Syntypen, Syrien, Libanon, durch KraAtz.

SDEI: 2 Syntypen, Syrien, durch KraAtz-Kasch., Coll. KraAtZ.

inaequalis Auriviluıus, 1908, Heteroclytomorpha

Dtsch. ent. Ztschr. 1908, 220, Taf. 3, Fig. 7

Mehrere Syntypen, Admiralitäts-Inseln, leg. v. BENNIGSEN.

SDEI: 1 Syntypus, Admiralitäts Inseln, Coll. v. BeNNIGSEN.

inderiense Suvorov, 1911, Compsodorcadion

Rev. Russe d'Ent. 11, 69-70

Mehrere Syntypen, Gebiet Uralsk, Indersk-See.

SDEI: 6 Syntypen, Indersk, Coll. KLÄGER.

inermis ScHWARZER, 1925, Ostedes

Ent. Bl. 21, 146-147

Mehrere Syntypen, Formosa: Fuhosho, 7.VII., 7.VIII., 7.IX.; Hoozan, 1908/V., IX.1910; Alikang, 7.X.

SDEI: Formosa, leg. SAUTER, Syntypen: 5 Ex. Fuhosho, 7.VIII., 7.IX.; 2 Ex. Hoozan, IX.1910/08-10; 1 Ex. Alikang, 7.X.

\section{infernale Mulsant \& Rey, 1863, Dorcadion}

Ann. Soc. Linn. Lyon (2) 10, 158-159

Mehrere Syntypen, Persien, Coll. Pellet.

SDEI: 1 Syntypus, Persia, Coll. KraATz.

\section{ingeae PEKs, 1993, Dorcadion}

Coleoptera, Schwanfelder Col. Mitt. 1, 3-5, Abb. 1-7

o Holotypus, Paratypen, Türkei (Edirne): sw Keşan, 150 m, 30.4.1992, leg. Peks, in Coll. Peks; Paratypen, sw Keşan, 150 m, 21.1.1992, leg. Rasse.

SDEI: 2 Paratypen, mit den Angaben des Holotypus.

insignis AuriviluIus, 1907, Obereopsis

Dtsch. ent. Ztschr. 1907, 84-85

Mehrere Syntypen, Togo, leg. Conradt, im SDEI.

SDEI: 1 Syntypus, Togo, leg. Conradt, Coll. KraAtz.

\section{insulare KrAAtz, 1873, Dorcadion}

In: KüsteR, Käf. Europ. 29, 62

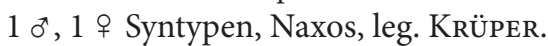

SDEI: 1 Syntypus, Naxos, 10.IV.1862, leg. Krüper, Coll. KrAATZ. 
irene GounelLe, 1911, Ophiostomis

Ann. Soc. ent. Fr. 80, 1-3

$9 \sigma^{*}, 4 o^{*}$ Syntypen, Brasilien, Prov. Goyaz, Jatahy.

SDEI: 2 Syntypen, Brésil, Prov. Goyaz, Jatahy, Coll. KraAtZ.

\section{jakovlevi Suvorov, 1912, Neodorcadion}

Rev. Russe d'Ent. 12, 70-71

Mehrere Syntypen, Alashan-Gebirge, Schlucht Chatengol, 5.-10.VI.1908, Exped. von Kozlov, Coll. SemenovTIAN-SHANSKY.

SDEI: 1 Syntypus, Gobi, Alashan, 12.VI.1908, leg. KozLOV, Coll. KLÄGER.

\section{jakovlevianum Suvorov, 1911, Compsodorcadion}

Rev. Russe d'Ent. 11, 60-61

Mehrere Syntypen, Chinesisches Gebiet östlich vom See Zaisan, Ort Mai-Kaptshagai, 1.VI.1903, leg. JAcoBson; Vorgebirge des Saikan, Bach Kalmak-pai, 2.VII.1900, leg. JACOBSON.

SDEI: 2 Syntypen, See Zaisan, Mai-Kaptshagai, 1.VI.1903, Kalmak-pai, 2.VII.1910 (nicht 1900!), leg. JACOBSON, Coll. KLÄGER.

\section{jakovlevianum zaisanense Suvorov, 1911, Compsodorcadion}

Rev. Russe d'Ent. 11, 61

Mehrere Syntypen, am südöstlichen Ufer des Zaisan Sees.

SDEI: 1 o $^{\star}$, Südost-Ufer des Sees Zaisan, 2.VI.1910, leg. Jacobson, Coll. v. Heyden; $2 \sigma^{*}$, Zaisan, 1.VI.1903, leg. GRUM: Syntypen.

\section{javeti KRAATZ, 1873, Dorcadion}

In: KüsteR, Käf. Europ. 29, 91

Mehrere Ex. Syrien, leg. Kindermann, in Coll. Javet und Coll. KraAtz; 1 Ex. Parnass, leg. KrüPer, in Coll. WiTTE: Syntypen.

SDEI: 1 Syntypus, Syrien, leg. Kindermann, Coll. KrAATZ.

\section{jordani HINTZ, 1911, Xylotrechus}

Wiss. Erg. Zentr. Afr. Exp. (1907/08) Zool. 3, 431

6 Syntypen, Congo Belge, Kondué, leg. Luja.

SDEI: 1 Syntypus, mit den Angaben der Beschreibung.

\section{kankauensis Schwarzer, 1925, Perissus}

\section{Ent. Bl. 21, 26-27}

Mehrere Syntypen, Formosa, Kankau, V., VII.1912, leg. SAUTER.

SDEI: 3 Syntypen, Formosa, Kankau, Koshun, V., VII. 1912, leg. SAUTER.

\section{kollari KRAATZ, 1873, Dorcadion}

In: KüsteR, Käf. Europ. 29, 87

Mehrere Syntypen, bei Amasia, leg. Kindermann, in Coll. KraATz, im k.k. Naturalienkabinet in Wien.

SDEI: 2 Syntypen, Amasia, Coll. KraATz.

\section{koltzei Heyden, 1887, Brachyta}

Dtsch. ent. Ztschr. 31, 304

i Holotypus, Amur, Nikolajevsk, leg. Graeser, durch Koltze, in Coll. v. Heyden, im SDEI.

\section{korostelevi DANILEVSKY, 1985 Agapanthia}

In: DAnilevsky M. L. \& Miroshnikov A. I. 1985, p. 385: Zhukidrovoseki Kavkaza (Coleoptera, Cerambycidae). Opredelitel. Krasnodar: Kubanskiy Selskokhozyaistvennyy Institut, 417 pp., 10 pls.

SDEI: $1 \sigma^{\star}, 1+$ Paratypen, Transcaucasia, Nakhichevan Buzgov, 7.06.1982, leg. Danilevky.

\section{kraatzi PIc, 1892, Stenopterus}

Echange 8, 21, 22, 23

Mehrere Syntypen, Asie mineure, Smyrne.

SDEI: 1 Syntypus, ohne Fundort, Coll. KraAtz.

\section{krueperi KraAtz, 1859, Acanthoderes}

Berl. ent. Ztschr. 3, 56-57, Taf. 3, Fig. 11

$10^{\star}, 1$ ㅇ Syntypen, Acarnanien, am See von Vrachori, leg. KRÜPER.

SDEI: Lectotypus, Graecia, leg. KrÜPER, Coll. KraAtZ, Designation: SAMA.

\section{kubanicum PLAVILSTSHIKOV, 1934, Dorcadion}

Ent. Bl. 30, 120-121, 122

Mehrere Syntypen; Caucasus bor., prov. Kuban, Maikop, 27.III.1912; st. Tichoretzkaja, 15.VII.1914; st. Labinskaja, 26.IV.1930; leg. DobrovlJAnski; st. Ladozhskaja, 3.V.1925; prope Labinskaja, 25.IV.1925; in Coll. PlaVILSTSHIKOV.

SDEI: 1 Syntypus, Caucasus bor., Maikop, 27.IV.1912 (nicht III.!).

\section{kurda Suvorov, 1913, Dorcadion}

Rev. Russe d'Ent. 13, 76

Mehrere Syntypen, im Karischen Gebiet, Sarakamysch. SDEI: 2 Syntypen, Kars., Sarakamysch, VI.1911, leg. Suvorov, Coll. KLÄGER.

\section{lacteomaculata ScHWARzER, 1925, Glenea}

Ent. Bl. 21, 151-152

Mehrere Syntypen, Formosa: Kosempo, V.1912, Sokutsu; 7.VI.1912, leg. SAUTER.

SDEI: Formosa, leg. SAUTER, Syntypen: 1 Ex. Sokutsu, 7.VI.1912; 1 Ex. Kosempo, 7.VI.1912.

\section{laevicollis ScHMIDT, 1922, Rhopalizus}

Arch. Naturg. 88, A 6, 94

Holotypus, Kamerun, leg. ConRADT, im SDEI.

\section{latefascia Schwarzer, 1925, Pterolophia}

Ent. Bl. 21, 65-66

Mehrere Syntypen, Formosa: Kosempo, 7.IX.1909; Kankau, IV.1912; leg. SAUTER.

SDEI: 2 Syntypen, Formosa: Kosempo, 7.IX.1909; Kankau, Koshun, IV.1912; leg. SAUter. 
lateralis AuniviLuIus, 1907, Blepisanis

Dtsch. ent. Ztschr. 1907, 85

Mehrere Syntypen, Togo, leg. Conradt, im SDEI.

SDEI: 1 Syntypus, Togo, leg. Conradt, Coll. KraAtz.

laterialba SCHWARzen, 1925, Albana

Ent. Bl. 21, 68

o Holotypus, Formosa, Anping, VII.1911, leg. SAUter, im SDEI.

\section{latericium GouneLLE, 1909, Periboeum}

Ann. Soc. ent. Fr. 77, 638-639

Mehrere Syntypen, Brasilien, Prov. Goyaz, Jatahy.

SDEI: 1 Syntypus, Brésil, Prov. Goyaz, Jatahy, Coll. KRAATZ.

laterinuda quadrivitticollis BREUNING, 1958, Glenea Beitr. Ent. 8, 494

ㅇ Holotypus, 1 Paratypus, Neu Guinea, leg. WAHnes, im SDEI durch FrankLin MÜLLER.

lativittis KraAtz, 1878, Dorcadion

Dtsch. ent. Ztschr. 22, 219-220

Mehrere Syntypen, Sibirien, Tarbagatai, leg. HaberHAUER.

SDEI: 4 Syntypen, Tarbagatai, leg. Haberhauer, Coll. F. MÜLLER.

\section{latula Broun, 1893, Somatidia}

Man. N. Zealand Col. 7, 1499

Mehrere Syntypen, New Zealand, Maketu.

SDEI: 3 Syntypen, Auckland, Maketu, Hunua, Coll. BROUN.

\section{lederi KRaAtz, 1878, Dorcadion}

Dtsch. ent. Ztschr. 22, 217-218

Mehrere Syntypen, Caucasus, leg. Leder.

SDEI: 8 Syntypen, Caukasus, leg. Leder, Coll. KraAtz, Coll. Kläger, Coll. Stierlin, Coll. F. MüLler.

\section{lederi KraATz, 1879, Fallacia}

Dtsch. ent. Ztschr. 23, 280

Mehrere Syntypen, Caucasus, Aksu, Borshom, Suram. SDEI: 5 Syntypen, Caucasus, leg. Leder. Coll. KraAtz.

\section{leucogrammum Suvorov, 1909, Neodorcadion}

Rev. Russe d'Ent. 9, 82-83

$3 \sigma^{*}, 1$ ㅇ Syntypen, nord-westl. Mongolei, nördl. Abhang des Gebirgsrückens Tanny-Ola, Anfang VIII.1903, Exped. von Grum-Grzhimailo, Coll. Semenov-TianSHANSKY.

SDEI: 2 o Syntypen, Geb. Tanny-ola, Kemtschik, 10.VIII. 1903, leg. Grum, Coll. KLÄGER.

libanoticum KraAtz, 1873, Dorcadion

In: KüsTER, Käf. Europ. 29, 100
Mehrere Syntypen; Syrien, durch Ehrenberg; Libanon, leg. Brûlerie; in Coll. Mniszech und im Museum Berlin.

SDEI: 1 Syntypus, Libanon, leg. BrûLERIE, Coll. KraAtz.

ligata Schwarzer, 1924, Cacia

Nova Guinea 15, 58

Mehrere Syntypen, N. West Neu-Guinea, Prauwenbivak, Idenburg River, 1920, leg. v. Heurn, Holländ. Exped.

SDEI: 1 Syntypus, mit den Angaben der Beschreibung, ex Museum Buitenzorg.

\section{lineatocolle KraAtZ, 1873, Dorcadion}

In: KüsTER, Käf. Europ. 29, 57; als var. von femoratum BRULLÉ beschrieben

Mehrere Syntypen, bei Saloniki, leg. Raymond, in Coll. v. Bruck, v. Kiesenwetter, Müller und KraAtz.

SDEI: 2 Syntypen, Saloniki, leg. Raymond, Coll. KraAtZ.

Lectotype, 1 Paralectotype, Designation: Sama.

\section{lineatocollis MeLzer, 1934, Odontocera}

Arch. Inst. biol. S. Paulo 5, 220-221, Taf. 15, Fig. 14

Holotypus, Brasilien, Estado de Santa Catarina, Teresopolis, im SDEI 1878, leg. FruHSTORFER.

longipillis PIC, 1898, Cortodera

Matériaux nour servir á l' étude des Longicornes, Heft 2, 50 Mehrere Syntypen, Syrie, leg. Leuthner.

SDEI: 12 Syntypen, Syrien, leg. LeuthNeR, durch Kraatz, Coll. v. Heyden, Coll. Kraatz.

\section{longissima AuRIVILLIUS, 1907, Oberea}

Dtsch. ent. Ztschr. 1907, 84

Mehrere Syntypen, Kamerun, leg. ConRADT, im SDEI. SDEI: 2 Syntypen, Kamerun, leg. Conradt, Coll. KraAtz.

lucae PIC, 1898, Compsodorcadion

Matériaux nour servir á l' étude des Longicornes, Heft 2, 42, 46

Syntypen, Kuldscha, in Coll. Heyden.

SDEI: 1 Syntypus, Kuldscha, Turkst. Staudgr. 85.

lugubre KRAATZ, 1873, Dorcadion

In: KüsTeR, Käf. Europ. 29, 41

Mehrere Syntypen; Macedonien, leg. v. Frivaldsszky; bei Salonick, leg. RaYmond, in Coll. v. Bruck, Coll. v. Kiesenwetter, Coll. Müller und Coll. Kraatz.

SDEI: 1 Ex. ohne Fundort, leg. v. Frivaldszky; 7 Ex. Salonick, Coll. Kratitz, Coll. F. MÜller, Coll. Stierlin: Syntypen.

\section{lujae HINTZ, 1911, Dityloderus}

Wiss. Erg. Dtsch. Zentr. Afr. Exp. (1907/08) Zool. 3, 433 5 Syntypen, Congo Belge, Kondué, leg. Luja, Exped. SDEI: 2 Syntypen, Congo Belge, Kondué, leg. LuJA. 
luteostriatus HeLleR, 1912, Tonesisternus

Ent. Mitt. 1, 172, Fig. 2

Mehrere Syntypen, Nova Guinea Germanica, Sattelberg, Coll. v. Bennigsen.

SDEI: 1 Syntypus, D. Neuguinea, leg. v. Bennigsen.

\section{luteovittigera PIC, 1906, Phytoecia}

Echange 22, 4

Mehrere Syntypen, Persien, Coll. v. Bodemeyer.

SDEI: 1 Syntypus, Persien, Sultanabad, leg. v. BoDEMEYER, durch LEONHARD.

\section{luteus australis HÜDEPOHL, 1979, Oxymerus}

Ent. Arb. Mus. G. Frey 28, 34, 36-37

$0^{\top}$ Holotypus, Argentina, Buenos Aires, Olivos, I.1977, in Coll. HÜDEPOHL; zahlreiche $o^{\star}$ ㅇ Paratypen, siehe Originalbeschreibung.

SDEI: 2 Paratypen, Argentin., Prov. Buen. Aires, 21.I. 1906, leg. BRUCH.

\section{luteus occidentalis HüDEPOHL, 1979, OXymerus}

Ent. Arb. Mus. G. Frey 28, 34, 36

o Holotypus, Bolivia, Region Chapare, 400 m, XII.1949, leg. ZischKa, in Coll. HüdepohL; zahlreiche ơ o Paratypen siehe Originalbeschreibung.

SDEI: 1 Paratypus, Peru.

\section{lutshniki PLAVILSTSHIKOV, 1937, Neodorcadion}

Acta Ent. Mus. Nat. Pragae 15, 33-34

Mongolie bor. occ.: république de Touva, vall. fl. Cha-kem, 19.VI., 5 ơ, 4 ㅇ durch Lutshnik, in Coll. Plavilstshikow; fl. Ulukem, village Urtshajlyk, 23. VI.1914 und fl. Cha-kem, 21.VI.1914, 13 o , 9 i leg. Tomashinsky, in Coll. Institut Zool. Acad. Sciences de l' URSS: Syntypen.

SDEI: 1 Syntypus, Tannu-tuva, vall. fl. Cha-kem, 23.VI. 1928.

Iydiae PLAVILSTSHIKOV, 1929, Dorcadion

Col. Centr.bl. 3, 209-212

$23 o^{\star}, 7$ i Syntypen, Sibiria mer. occ., Prov. Semipalatinsk, nahe Semipalatinsk, 12., 13., 14.V.1927, Dzhana-Semej, 10.V.1928, leg. Altynbajev, in Coll. Plavilstshikov. SDEI: 1 Syntypus, Sibiria mer., occ., Prov. Semipalatinsk, 12.V.1927, leg. Altynbajev.

\section{maaki KraAtz, 1879, Callidium}

Dtsch. ent. Ztschr. 23, 106-107

Holotypus, Ost-Sibirien, Amur, leg. CHrIstoph, im SDEI 1877, Coll. KraAtz.

\section{macilentum KraAtz, 1879, Microrhabdium}

Dtsch. ent. Ztschr. 23, 99-100, Taf. 1, Fig. 8

Holotypus, Ost-Sibirien, Amur, leg. ChrIstoph, im SDEI 1877, Coll. KraATz.

macropus KraATZ, 1873, Dorcadion

In: KüsteR, Käf. Europ. 29, 99
Mehrere Syntypen, Amasia, leg. Lederer, in Coll. KraAtz und Coll. MNiszech.

SDEI: 2 Syntypen, Amasia, leg. Lederer, Coll. KraAtz.

maculata SCHWARZER, 1925, Xoanodera

Ent. Bl. 21, 21

Mehrere Syntypen, Formosa, Sokutsu, 7.VI.1912, leg. SAUTER.

SDEI: 3 Syntypen, Formosa, Banshoryo Distr., Sokutsu, 7.VI., 7.VII.1912, leg. SAUTER.

\section{maculatus Hintz, 1913, Pinacosternodes}

Dtsch. ent. Ztschr. 1913, 199

Mehrere Syntypen, Congo-Belge, Kondué.

SDEI: 1 Syntypus, Congo-Belge, Kondué, leg. LujA, Coll. KrAATZ.

madoni PIC, 1890, Clytus

Bull. Soc. ent. Fr. 1890, 211

Mehrere Syntypen, Palestine, leg. MADoN.

SDEI: 1 Paralectotypus, Palestine, Coll. v. Heyden, Designation: SAMA.

\section{magnifica SchWARzER, 1925, Saperda}

Ent. B1. 21, 148

Mehrere Syntypen, Formosa, Kosempo, 7.VI.1912, leg. SAUTER.

SDEI: 1 Syntypus, Formosa, Kosempo, 7.VI.1912, leg. SAUTER.

\section{mali HolzschuH, 1993, Rhopalopus}

FBVA-Berichte-Schriftenreihe der forstl. Bundesanstalt in Wien 75: 34

SDEI: 2 + Paratypen, Kirgistan, Chatkalskiy Mts. 1900 m, Sary-chelek Nat. Reserve, 10.-11.07.1991, leg.

O. Ment; Kirgistan, Dsjala-Abad Town, 18.07.1991, leg. O. MEHL.

\section{malthinoides KraAtz, 1879, Pseudopidonia}

Dtsch. ent. Ztschr. 23, 104, Taf. 1, Fig. 11; als var. von alticollis KraAtz beschrieben

Holotypus, Ost-Sibirien, Amur, leg. Christoph, im SDEI 1877, Coll. KraAtz.

marcae LóPEz-CoLón, 1997 Plagionotus

Lambillionea 97: 221-224

○ Holotypus, Espana, Rivas-Vaciamadrid, Covibar, 12.VII.1993, leg. \& Coll. López-Colón; 259 Paratypen siehe Originalbeschreibung.

SDEI: 1 Paratypus, Espana, Rivas-Vaciamadrid, 9.VIII. 1991, leg. LóPEZ-CoLóN.

\section{mardinensis Heyden, 1894, Phytoecia}

Dtsch. ent. Ztschr. 1894, 87-88

o Holotypus, Asia minoris, Mardin, in Coll. Heyden. SDEI: Holotypus, Asia min., Kastomuni, PrEIss. 
marginatus SCHWARZER, 1925, Olenecamptus

Ent. Bl. 21, 63-64

Mehrere Syntypen, Formosa, Kosempo, 7.VII., leg. SAUTER.

SDEI: $10^{\Uparrow}$ Syntypus, Formosa, Kosempo, 7.XII. (nicht VII.!).

\section{marianarum AuRIVILLIUS, 1908, Monochamus}

Dtsch. ent. Ztschr. 1908, 216

Mehrere Syntypen, Marianen-Inseln, leg. v. Bennigsen. SDEI: 11 Syntypen, Marianen, Coll. v. Bennigsen und Coll. KraAtz.

\section{marianarum AuriviLlius, 1908, Prosoplus}

Dtsch. ent. Ztschr. 1908, 222

Mehrere Syntypen, Marianen-Inseln, leg. v. Bennigsen. SDEI: 2 Syntypen, Marianen, Coll. v. Bennigsen und Coll. KraAtz.

\section{marmorata GAHAN, 1898, Prosopocera}

Ann. Mag. Nat. Hist. (7) 2, 48

Mehrere Syntypen, Brit. E. Africa, Samburu, leg. Betton. SDEI: 1 ơ $^{\star} 1$ क Syntypen, Samburu, 30.X.-20.XI.1896, Coll. KraAtz.

marmorata AuRIVILLIUS, 1908, Trigonoptera

Dtsch. ent. Ztschr. 1908, 221, Taf. 3, Fig. 6

Mehrere Syntypen, Deutsch Neu-Guinea, Simbang und Sattelberg, leg. v. BENNigsen.

SDEI: 2 Syntypen, D. N. Guinea, Sattelberg, Coll. v. BENNIGSEN, Coll. KraATZ.

\section{merceti Schramm, 1910, Dorcadion}

Bol. Real. Soc. Esp. Hist. Nat. 10, 286-287

Mehrere Syntypen, Las Rozas, Sierra de Gredos, durch ARDoIs.

SDEI: 6 Syntypen, Las Rozas, Gredos, durch Ardois, Coll. KLÄGER.

mingrelica KraAtz, 1879, Fallacia

Dtsch. ent. Ztschr. 23, 279-280

Holotypus, Mingrelien, leg. Haberhauer (Gurien), in Coll. KraAtZ, im SDEI.

\section{minutum KraAtz, 1873, Dorcadion}

In: KüsteR, Käf. Europ. 29, 76

Mehrere Syntypen, Griechenland, bei Athen, leg. v. Heldreich und KRÜPER.

SDEI: 6 Syntypen, Graecia, Coll. KraATz (als ab. irroratum KraATz bezettelt).

\section{mirabilis KraAtz, 1896, Cyrtocerus}

Dtsch. ent. Ztschr. 1896, 155-156, Fig.

o Holotypus, Africa or., Mpuapua, in Coll. KraAtz, im SDEI.

miraculum ReITTER, 1897, Neodorcadion

In: KARsCH, Ent. Nachr. 23, 182-183
Mehrere Syntypen, Mongolei, Changai-Gebirge, leg. LEDER.

SDEI: 1 Syntypus, nördl. Mongolei, Changai, leg. LEDER, Coll. v. Heyden.

moesiaca DANIEL, 1891, Leptura

Col. Stud. 1, 6, 27-28

Mehrere Syntypen; Serbien, leg. Zebe, Coll. KraAtz; Türkei, im Ung. National-Museum; Balkan, leg. v. FrIvALDSZKY, Coll. BAUDI.

SDEI: 5 Ex. Serbien, leg. Zebe; 1 Ex. ohne Fundort, leg. v. Frivaldszky; Coll. KraAtZ: Syntypen.

moisescoi LePESME, 1946, Corus

Bull. Soc. ent. Fr. 51, 137-138

Holotypus, Usambara, leg. WEISE, im SDEI (Ex. ohne caput).

morio KRAATZ, 1879, Necydalis

Dtsch. ent. Ztschr. 23, 106

q Holotypus, Ost-Sibirien, Amur, leg. Christoph, im SDEI 1877, Coll. KraATZ.

morio KrAATZ, 1879, Oberea

Dtsch. ent. Ztschr. 23, 117

o Holotypus, Ost-Sibirien, Amur, leg. Christoph, im SDEI, Coll. KraATZ.

morozovi DANILEVSKY, 1992, Dorcadion

Lambillionea 92: 94-95

o Holotypus, Kazakhstan, Charyn valley 1300 m, 26 km to the North from Zhalanash, 15.IV.1991, leg. \& Coll. DANILEVSKY; mehrere Paratypen siehe Originalbeschreibung. SDEI: 2 क Paratypen, Kazakhstan, 26 km N Zhanlanash, 1300 m, 15.IV.1991, leg. M. DANILEvsky.

muchei plurivittipenne BREUNING, 1962, Dorcadion

Reichenbachia 1, 38

ㅇ Holotypus, Anatolien, Cankiri, IV.1961, leg. Muche, in Coll. Muche; 3 Paratypen, dito.

SDEI: 2 Paratypen, Anatolia, Cankiri, IV., leg. Muche.

muliebris HEYDEN, 1886, Leptura

Dtsch. ent. Ztschr. 30, 276

2 Syntypen, Amur, Suyfun-Fluß, durch Staudinger, No. 383 , in Coll. v. Heyden, im SDEI.

mulsanti STIERLIN, 1866, Molorchus

Mitt. Schweiz. ent. Ges. 2, 30-31

Syntypen. Sicilien.

SDEI: o Lectotypus, 1 + Paralectotypus, Sicil., STIERLIN; 1 o Paralectotypus, Sicilien, STIERLIN; Designation: SAMA.

mysticus Melzer, 1932, Pentheochaetes

Rev. Ent. S. Paulo 2, 433-434, Taf. 7, Fig. 8

Brasilien, Estado de São Paulo, Capital, leg. Metz; Bosque da Saude perto da Capital, leg. Melzer; Estado 
do Paraná, Rio Negro, leg. Franciscanos: Holotypus, mehrere Paratypen, im SDEI und in Coll. MeLzer.

SDEI: Holotypus, Santos; 2 Paratypen, Sao Paulo, leg. METZ.

\section{nais GounELLE, 1911, Ommata}

Ann. Soc. ent. Fr. 80, 28-29

7 o 3 i Syntypen, Brasilien, Prov. Goyaz, Jatahy.

SDEI: $1 \sigma^{\star}$ Syntypus, mit den Angaben der Beschreibung.

\section{namaganensis HEYDEN, 1885, Clytus}

Dtsch. ent. Ztschr. 29, 297-298

4 Syntypen, Turkestan, Namagan, durch STAudinger, in Coll. v. Heyden.

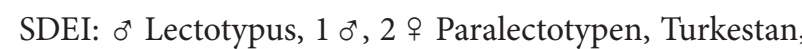
Namagan, durch STAudinger, Designation: SAma.

\section{neglecta Melzer, 1932, Oreodera}

Rev. Ent. S. Paulo 2, 425-426, Taf. 6, Fig. 3

Holotypus, Brasilien, Estado de Para /Mathan), im SDEI.

neglectum GouneLLE, 1911, Callichroma

Ann. Soc. ent. Fr. 80, 70, Fig.

8 o Syntypen, Brasilien, État de Goyaz, Reg. Jatahy, im Museum Paulista, SDEI und in Coll. GounELle.

SDEI: 1 Syntypus, Brésil, Ét. de Goyaz, Jatahy.

niger GRESSITT, 1984, Tmesisternus

Pacific Insects Monogr. 41, 106-107, Taf. 6 i

$0^{*}$ Holotypus, PNG, New Guinea, E Highlands, Okapa, 1700-2000 m, 19.I.1966, leg. SEdLACEK, im Bishop Mus. Honolulu; Paratypen siehe Originalbeschreibung. SDEI: 4 Paratypen, D. Neu Guinea, Wahnes, Franklin MÜLLER.

nigra AURIVILLIUS, 1907, Syndere

Dtsch. ent. Ztschr. 1907, 78-79

Mehrere Syntypen, Kamerun, leg. Conradt, via SCHENKLING an AURIVILLIUS, im SDEI.

SDEI: 1 Syntypus, Kamerun, leg. Conradt, Coll. KraAtz.

nigra KiESENWETTER, 1859, Tetrops

Berl. ent. Ztschr. 3, 57

Mehrere Syntypen, Sardinien, durch GHILIANI.

SDEI: Lectotypus, Sardinien, durch Ghiliani, Coll. KraAtz, Designation: SAma.

nigrita HEYDEN, 1876, Cortodera

Dtsch. ent. Ztschr. 20, 318

+ Holotypus, Hungaria, Dahl.

nigritulus KraATz, 1879, Clytus

Dtsch. ent. Ztschr. 23, 109-110

Mehrere Syntypen, Ost-Sibirien, Amur, leg. Christoph. SDEI: 3 Syntypen, Amur, 1877, leg. Christoph, Coll. KraATZ. nigrofasciatus AuRIVILLIUS, 1907, Leiopus

Dtsch. ent. Ztschr. 1907, 82

Mehrere Syntypen, Kamerun, leg. ConRAdT, im SDEI. SDEI: 1 Syntypus, Kamerun, leg. ConradT, Coll. KraAtZ.

nigrofasciatus AuriviluIus, 1908, Tmesisternus

Dtsch. ent. Ztschr. 1908, 214, Taf. 3, Fig. 8

Mehrere Syntypen, Deutsch Neu-Guinea, leg. v. BeNNIGSEN.

SDEI: 1 Syntypus, D. Neu Guinea, Coll. v. Bennigsen.

nigrotriangularis HELLER, 1914, Tmesisternus

Dtsch. ent. Ztschr. 1914, 316

Mehrere Syntypen, Nova Guinea Germanica, ex Coll. v. Bennigsen, im Museum Dresden und SDEI.

SDEI: 1 Syntypus, N. Guinea, Sattelberg, leg. und Coll. v. BeNNigsen.

nitida AurIVILLIUS, 1907, Sophronica

Dtsch. ent. Ztschr. 1907, 81

Mehrere Syntypen, Kamerun, leg. ConRAdT, im SDEI. SDEI: 1 Syntypus, Kamerun, leg. ConradT, Coll. KraAtZ.

notula GounELLE, 1909, Eburodacrys

Ann. Soc. ent. Fr. 77, 632

4 Syntypen, Brasilien, Prov. Goyaz, Jatahy. SDEI: 1 Syntypus, Brésil, Prov. Goyaz, Jatahy, Coll. KraAtz.

nuristanicus Heyrovsky, 1936, Purpuricenus

Arb. morph. tax. Ent. 3, 212-213

2 ơ $^{\star} 3$ ㅇ Syntypen; Nuristan, Gulan-Tal, 28.VI.; Brubrutz im Kti-Tal, 18.VI.; Hindukusch-Expedit., 1935; im SDEI und in Coll. Heyrovsky.

SDEI: 3 Syntypen, Afghan., Nuristan; Gulan-Tal, 28.VI. 1935; b. Brubrutz im Kti-Tal, 18.VI.1935; Expedit. SCHEIBE (2 Ex. als f. bisignata und f. rubripennis HeYrovsky bezettelt).

nivipictus KraATz, 1879, Clytus

Dtsch. ent. Ztschr. 23, 91 nata

Syntypen, Cilicischer Taurus, Kisbek, leg. LEDER.

SDEI: Lectotypus „Külek“, Designation: SAMA.

obscura Schwarzen, 1925, Abryna

Ent. Bl. 21, 65

Mehrere Syntypen, Taihorin, 7.VIII.1911, leg. SAUTER. SDEI: 1 Syntypus, Formosa, Taihorin, 7.VIII.1911, leg. SAUTER.

\section{obscura Schwarzer, 1925, Pterolophia}

Ent. Bl. 21, 66-67

Mehrere Syntypen, Formosa, leg. SAUTER.

SDEI: 3 Ex. Taihorin, 7.VII., 22.VIII., 7.XI.1911; 13 Ex. Sokutsu, Banshoryo Distr., VI., 7.VI., 22.VI., 7.VII., 7.VIII.1912; 2 Ex. Alikang, 7.IX., 7.X.; 9 Ex. Kankau, Kos- 
hun, IX.1912; 41 Ex. Kosempo, 7.IX.1909, 7.XII.1909/ 7.VII., 7.IX., 7.X., 7.XII.1911, 22.V.1912, VI.1912, 22.VII. (?); 1 Ex. Suisharyo, 7.XII.1911; 1 Ex. Hoozan, 7.IX.; 1 Ex. Fuhosho, VII.1909; Formosa, leg. SAuter, Syntypen.

\section{obsoletum KraATz, 1973, Dorcadion}

Käfer Europas, 29. Heft: 78

Mehrere Syntypen, Brussa, Coll. KLÄGER, Coll. KraAtz. SDEI: Lectotypus, 5 Paralectotypus, Brussa, Coll. KraAtZ, KLÄGER, Designation: Sama.

ocellatum GouneLLe, 1909, Periboeum

Ann. Soc. ent. Fr. 77, 640

Mehrere Syntypen, Brasilien, Prov. Goyaz, Jatahy.

SDEI: 1 Syntypus, Brésil, Prov. Goyaz, Jatahy.

\section{ochraceipennis KraAtz, 1882, Phytoecia}

Dtsch. ent. Ztschr. 26, 337

Mehrere Syntypen, Samarkand, leg. Haberhauer.

SDEI: 1 Syntypus, Samarkand, 1881, durch STAUdINGER, Coll. KraAtz.

oertzeni GANGLBAUER, 1884, Dorcadion

Verh. Zool. Bot. Ges. Wien (1883) 33, 454

Mehrere Syntypen, Attika.

SDEI: 2 Syntypen, Attika, durch Ganglbauer, Coll. KOLTZE.

olivacea AuriviluIus, 1908, Trigonoptera

Dtsch. ent. Ztschr. 1908, 221, Taf. 3, Fig. 4

Mehrere Syntypen, Deutsch Neu-Guinea, Simbang, Sattelberg, Herbertshöhe, leg. v. BENNIGSEN.

SDEI: 1 Ex. D. N. Guinea, Herbertshöhe; 1 Ex. N.-Guinea, Sattelberg; leg. v. Bennigsen, Coll. v. Bennigsen, Syntypen.

\section{olympicum flavosuturale KRÄTSCHMER, 1987}

\section{Dorcadion}

Ent. Ztschr. 97, 340

$\sigma^{*}$ Holotypus, $30^{*} 4$ Paratypen, Türkei, Anatolien occ., Ufer Uluabat Göl, IV.1979, leg. CzIPKA, in Coll. Krätschmer; Paratypen, dito 7.IV.1986 und 19.IV.1987, in Coll. Drovenik, Peks und Czipka.

SDEI: 2 Paratypen, TR, Vil. Bursa, Ufer Uluabat Göl, 19.IV.1987, leg. DroveniK.

optatum toropyginae DANILEVSky, 1999, Dorcadion

Coléoptères 5: 22-23

$0^{*}$ Holotypus, Kirgizia, left side of the Ala-Archa Narrows, $20 \mathrm{~km}$ S Bishkek, $1800 \mathrm{~m}, 2$. V.1997, leg. \& Coll. DANILEVsky; 344 Paratypen siehe Originalbeschreibung. SDEI: 2 o $^{\top}$ Paratypen, Kirgizia, Ala-Archa, 1700 m, 3.-4.V.1997, leg. M. Danilevsky.

orientalis natalensis FIEDLER, 1938, Ceroplesis

Dtsch. ent. Ztschr. 1938, 605
Holotypus, 43 Paratypen, Afrika, Natal, im Museum Berlin, Museum Brüssel, Senckenberg Museum und SDEI.

SDEI: 1 Paratypus, Natal, Coll. Franklin Müller.

\section{orientalis pseudocaffer FIEDLER, 1938, Ceroplesis}

Dtsch. ent. Ztschr. 1938, 605

Holotypus, 51 Paratypen, Deutsch-Südwestafrika, Windhuk, Okahandja, Malakong, Lino Kana, Gaberones, Matabele, Kubub, Warmbad; im Museum Berlin, Museum Brüssel, Senckenberg Museum, Museum Paris und SDEI.

SDEI: 2 Ex. Südafrika, Warmbad, VI.1912, det. FRIEdRICHS; 1 Ex. DSWA, Kubub, Coll. v. Bennigsen: Paratypen.

\section{ottomanum HEYDEN, 1863, Brachyteroma}

Berl. ent. Ztschr. 7, 128-129, Taf. 4, Fig. 4

o Holotypus, Türkei, durch Michahelles, im SDEI, Coll. v. Heyden.

\section{palimnoides SchWARzER, 1925, Apalimna}

Ent. Bl. 21, 62-63

Mehrere Syntypen, Formosa; Fuhosho, VI.1909; Hoozan, 7.IX.1910; Kosempo, 7.VII.1909/ 22.V.1912; Taihorin, 7.VII.1911; leg. SAUTER.

SDEI: Formosa, leg. Sauter, Syntypen: 9 Ex. Fuhosho, VI., VII.1909; 3 Ex. Kosempo, 7.VII.1909/ 7.VII.1911/ 22.V.1912; 2 Ex. Hoozan, 7.IX.1910; 1 Ex. Taihorin, 7.VIII.1911.

\section{pallipennis HEYDEN, 1887, Gymnopterion}

\section{Dtsch. ent. Ztschr. 31, 321-322}

2 o $^{\star}, 2$ ㅇ Syntypen, Turkestan, Issik-kul, Coll. v. Heyden. SDEI: Lectotypus, 2 Paralectotypen, Issik-kul, durch Staudinger und Krieghoff, Coll. v. Heyden, Designation: SAMA.

\section{papuanus HelleR, 1914, Coptocercus}

Dtsch. ent. Ztschr. 1914, 314-315, Taf. 12, Fig. 6

Mehrere Syntypen, Nova Guinea Germanica, Sattelberg, ex Coll. v. Bennigsen, im Museum Dresden und SDEI.

SDEI: 1 Syntypus, N.-Guinea, Sattelberg, leg. v. BENNIGSEN.

\section{pelionense BREIT, 1923, Dorcadion}

Wien. ent. Ztg. 40, 145-146

Mehrere Syntypen, Balkan, Gipfelregion des Pelion in Thessalien, 2. Hälfte IV., leg. MoczArsKi und BreIt. SDEI: 2 Syntypen, Thessalien, Pelion.

\section{peregrina KoLBE, 1900, Mecothyrea}

Berl. ent. Ztschr. 45, 302

1 Ex. West-Afrika, Mhonda; mehrere Ex. Mrogoro, Usegua, Usaramo, in Coll. v. Bennigsen: Syntypen. SDEI: 2 Syntypen, Usegua, Coll. v. BENNIGSEN. 
pfisteri StienLIN, 1864, Hammaticherus

Berl. ent. Ztschr. 8, 152

Mehrere Syntypen, Sicilien.

SDEI: 2 Syntypen, Sicilien, Coll. STIERLIN.

\section{philemon AdlBauer, Pachydissus}

Coleoptera, Schwanfelder Coleopterologische Mitteilungen 6: 169-171

Holotypus $\sigma^{\star}$, Zimbabwe, Hunyani river, S-Rhodesia, 15.VIII.1971, From Msasa Stump, A. J. Duke in Transvaal Museum, Pretoria; Paratypen siehe Originalbeschreibung.

SDEI: 4 Paratypen, Dar-es-Salaam, leg. und Coll. v. BENNigSen, Coll. KraAtz.

\section{pilosellum KraAtz, 1873, Dorcadion}

In: Küster, Käf. Europ. 29, 58

Mehrere Syntypen, Mytilene, durch FaIrmaire.

SDEI: 2 Syntypen, Mytilene, durch Faimaire, Coll. KraAtZ, Coll. KLÄGER.

\section{pilushana SaITo, 1979, Pidonia}

Bull. Nat. Sci. Mus. Ser. A (Zool.) 5 (3), 211-214, Fig. 1-6, 13 A-I $\sigma^{*}$ Holotypus, Pilu-shenmu, nahe Mt. Pilushan, $2.440 \mathrm{~m}$ alt., Hwalien Hsien, 8.V.1977, leg. SAIto; $3510^{*}$, 217 \& Paratypen, dito, Anfang V.-Anfang VI.1977 und 1978, leg. Fujita, Itoh, KubOKI, NiISATo, OdA, SAKaino, Shimomura und SAito; im National Science Museum (Nat.Hist.), Tokyo, in Coll. SAIto u. a. Coll.

SDEI: 2 Paratypen, Pilu-shenmu, Hwalien Hsien, Taiwan, 16.V.1978, leg. Shimomura und 4.V.1978, leg. Saito.

\section{piochardi KraATZ, 1873, Dorcadion}

In: KüstER, Käf. Europ. 29, 85

Mehrere Syntypen, asiatische Türkei, bei Samsun, Amasia, auf dem Ak Dagh Gipfel bei Cadik, leg. Lederer und leg. Kindermann.

SDEI: 1 Syntypus, ohne Fundort, Coll. KraATz.

\section{plagiata AuriviluIus, 1911, Androeme}

Arkiv för Zool. 7 (1), 4

Mehrere Syntypen, D. Ostafrika, Usambara, im SDEI und im Museum Stockholm.

SDEI: 1 đ Syntypus, Usambara, leg. Weise, Coll. KraAtz.

\section{plasoni GanglBauer, 1884, Dorcadion}

Verh. Zool. Bot. Ges. Wien 33 (1883), 491

Mehrere Syntypen, Persien, durch KraAtz und Plason und 1 Ex. Caspisches Meer, bei Rasano, leg. Leder; Syntypen.

SDEI: 4 Syntypen, Persien, Coll. KraAtz.

plasoni Ganglbauer, 1884, Phytoecia

Verh. Zool. Bot. Ges. Wien 33 (1883), 571

Mehrere Syntypen, Persien.

SDEI: 1 Syntypus, Persia, leg. Plason, Coll. KraAtz. prasiniventre GOUNELLE, 1911, Orthoschema

Ann. Soc. ent. Fr. 80, 76, nota

2 ơ, 3 ㅇ Syntypen, Brasilien, Prov. Goyaz, Jatahy, Espirito Santo, Bahia, im SDEI und in Coll. Gounelle.

SDEI: 3 Syntypen, Brasilia, Bahia, leg. Fruhstorfer, Coll. KraAtz.

\section{preissi HeYdEN, 1894, Dorcadion}

Dtsch. ent. Ztschr. 38, 86-87, Taf. 1, Fig. 3

$1 \sigma^{\star}, 1$ \% Syntypen, Asia minor, Kastamuni, durch PreIss, in Coll. v. Heyden; $10^{\star}$, dito, in Coll. Preiss.

SDEI: 2 Syntypen, mit den Angaben der Beschreibung.

preissi Heyden, 1894, Hesperophanes

Dtsch. ent. Ztschr. 1894, 85-86

\% Holotypus, Asia minor, Mardin, im SDEI durch STAUDINGER.

\section{prolixus Melzer, 1931, Ozineus}

Rev. Ent. S. Paulo 1, 7-8

11 Syntypen; Estado de São Paulo, Sao Sebastião, leg. BArbiellini; Esatado da Bahia, São Salvador, leg. BonDAR; Estado de Pernambuco, Tapéra, leg. Pickel.

SDEI: 1 Syntypus, Pernambuco, Tapéra, 21.IX.1928, leg. PICKEL.

\section{proximus Melzer, 1934, Gepturges}

Rev. Ent. Rio de Janeiro 4, 100

Holotypus, 1 Paratypus, Rep. Costa Rica, Hamburgfarm, Reventazon, Ebene Limon, leg. Nevermann, Turrialba, 800 m, leg. Schild, in Coll. Nevermann und Coll. MelZER.

SDEI: 1 Paratypus, Costa Rica, Turrialba, 800 m, leg. Schild, Coll. Nevermann.

\section{pseudomophlus ReITTER, 1899, Cortodera}

Dtsch. ent. Ztschr. 33, 40-41

Mehrere Syntypen, Araxesthal, bei Ordubad, leg. KuBISCHTEK.

SDEI: 5 Syntypen, Caucasus, Araxesthal, Coll. KraAtz, Coll. Stierlin, Coll. F. Müller.

\section{pseudosulcatus Schwarzer, 1924, Tmesisternus}

Nova Guinea 15, 59

Mehrere Syntypen, N. West Neu-Guinea, Pionierbivak, VII.-VIII.1920, leg. v. HeUrN.

SDEI: 2 Syntypen, N. N. Guinea, Pionierbivak, VII.VIII.1920, leg. v. Heurn, ex Museum Buitenzorg.

\section{pulchra SCHWARZER, 1925, Neoserixia}

Ent. Bl. 21, 148-149

Mehrere Syntypen, Formosa: Kankau, IV., V., VI.1912; Banshoryo, V.1912; leg. SAUter.

SDEI: Formosa, leg. SAuter, Syntypen: 8 Ex. Kankau, 7.IV., V., VI.1912; 1 Ex. Banshoryo, Shisha, V.VII.1912. 
pumilio PLAVILSTSHIKOV, 1951, Dorcadion

Sborn. trud. Zool. Mus. M. G. U. 7, 115

Mehr als 50 Syntypen, Kasachstan, Alma Ata Gebiet, Vorberge am Mittellauf des Ili, am häufigsten bei der Station Tschu; Kirgisien Frunse.

SDEI: 1 Syntypus, Dzhungar ross., St. Tshu, 27.IV.1934.

\section{punctata SCHWARZER, 1925, Batocera}

Ent. B1. 21, 60

Mehrere Syntypen, Formosa, leg. SAUter.

SDEI: 1 Syntypus, Formosa, Kosempo 7.VII.1911, leg. SAUTER.

\section{purkynei HeYRovsky, 1925, Dorcadion}

Acta Soc. Ent. „ech. 22, 45, Fig. 1, 2

Mehrere Syntypen, Mazedonien, Kajmakčalanu, 2000$2500 \mathrm{~m}$.

SDEI: 2 Syntypen, Macedonia, Kajmakčalanu, 20.VII. 1925.

pusilla AuriviLluUs, 1907, Stathmodera

Dtsch. ent. Ztschr. 1907, 80-81

Mehrere Syntypen, Kamerun, leg. ConRADT, im SDEI.

SDEI: 1 Syntypus, Kamerun, leg. Conradt, Coll. KRAATZ.

pygmaeum GanglBaUeR, 1881, Rhagium

Verh. Zool. Bot. Ges. Wien 31, 718

Mehrere Syntypen, Caucasus.

SDEI: 1 Syntypus, Caucasus, durch Ganglbauer, Coll. Koltze.

quadricostulatum KraAtZ, 1879, Megasemum

Dtsch. ent. Ztschr. 23, 98

2 Syntypen, Ost-Sibirien, Amur, leg. Christoph, im SDEI 1877, Coll. KraAtZ.

quadrimaculata NonfRIED, 1894, Triginoptera

Dtsch. ent. Ztschr. 1894, 211

Mehrere Syntypen, N. O. Sumatra, Tebing-Tinggi, leg. Schultheiss, in Coll. Schultheiss.

SDEI: 1 Syntypus, mit den Angaben der Beschreibung, 9.IV.1884.

quadrimaculatus Auriviluius, 1908, Tmesisternus

Dtsch. ent. Ztschr. 1908, 214-215, Fig. 10

Mehrere Syntypen, Herbertshöhe, leg. v. Bennigsen.

SDEI: 1 Syntypus, D. N. Guinea, Herbertshöhe, Coll. v. BENNIGSEN.

\section{quadrinotata SCHWARzER, 1921, Sybra}

Ent. Bl. 21, 145

Mehrere Syntypen, Formosa: Kosempo, 7.VII.1909/ 7.VII.1911; Kankau, V., VII.1912; leg. SAUTeR.

SDEI: Formosa, leg. SAUter, Syntypen: 3 Ex. Kankau, Koshun, V., VII.1912; 1 Ex. Kosempo, 7.VII.1911. quadriplagiata HINTZ, 1911, Antennoeme

Wiss. Erg. Zentr. Afr. Exped. Zool. 3, 427

6 Syntypen; Mawambi am Ituri, westl. v. Albert-See, IV.1908; Kondué, Congo Belge, leg. Luja.

SDEI: 2 Syntypen, Congo-Belge, Kondué, leg. LujA.

quadripustulatum KraATZ, 1873, Dorcadion

In: Küster, Käf. Europ. 29, 88

Holotypus, Dardanellen, im SDEI (ohne Fundort), Coll. KraAtz.

quadrisignatus SCHWARzeR, 1925, Micromulciber

Ent. Bl. 21, 64

Mehrere Syntypen, Formosa: Kosempo, 7.VII.; Sokutsu, 7.VII.1912; leg. SAUTER.

SDEI: 2 Syntypen, Formosa, Kosempo, 7.VI., 7.XII.1912, leg. SAUTER.

\section{ramalium BedeL, 1885, Pseudomyrmecion}

Bull. Soc. ent. Fr. (6) 5,132

Mehrere Syntypen, Algérie, Wald von Edough, 15.V.VII.

SDEI: 1 Syntypus, Edough, Coll. STIERLiN.

reini HEYDEN, 1879, Toxotus

Dtsch. ent. Ztschr. 23, 359-360; als var. von minutus GeBLER beschrieben

Holotypus, Japan, bei Osaka, leg. ReIn, im SDEI.

ressli Demelt, 1963, Oberea

Ent. Bl. 59, 150-151, Abb. 5

Holotypus, mehrere Paratypen, Asia minor sept., Kizilcahamam, leg. Ressl, in Coll. Demelt, Coll. Schurmann, Coll. Heyrovsky und Coll. Lekes.

SDEI: 1 Paratypus, Asia minor, Kizilcahamam.

ribbei KraAtz, 1878, Dorcadion

Dtsch. ent. Ztschr. 22, 220-221

Mehrere Syntypen, Sibirien, Tarbagatai.

SDEI: 4 Syntypen, Tarbagatei, leg. Christoph, durch Ribbe, Coll. Kraatz, Coll. v. Heyden.

richteri BRucH, 1908, Trachyderes

Rev. Mus. La Plata 15, 215-216

Mehrere Syntypen, Argentinien, Provincia, Catamarca, Andalgala, III.1907, durch VENTURI.

SDEI: 1 Syntypus, Argentinien, Prov. Catamarca, 29.III. 1907, durch Bruch, Coll. KraAtz.

ritae SAMA, 1985, Exocentrus

Lavori Soc. Ven. Sc. Nat. 10, 65-67

○ Holotypus, Turchia, Vil. Içel: Çamliyayla (Namrun), 1300 m, 31.V.81, leg. SAma, im Mus. civ. Stor. nat. Verona. Zahlreiche Paratypen siehe Originalbeschreibung.

SDEI: 1 Paratypus, Turchia-Namrun, Meselik, ex larva Morus, 30.VI./ 24.VII.81, leg. SAMA. 
riveti Gounelle, 1910, Mecometopus

Bull. Mus. Paris 16, 16

Holotypus, Équateur, Santo Domingo de Los Colorados, alt. 510 m, 1905, leg. Reveti; 3 Paratypen, Columbien, Rio Dagua, im SDEI und in Coll. Gounelle (als var. pilosa Gounelle beschrieben).

SDEI: $1 \sigma^{\star}, 1$ ㅇ Paratypen, Colombie, Coll. KraAtz (als var. pilosa GounelLe bezettelt).

\section{robustum GanglBauer, 1884, Dorcadion}

Verh. Zool. Bot. Ges. Wien 33 (1883), 500

o Holotypus, Amasia, durch Kraatz, im SDEI (ohne Fundort), Coll. KraAtz.

\section{robustus HeYDEN, 1844, Aulacopus}

Stett. ent. Ztg. 5, 15

Mehrere Syntypen, europäische Türkei.

SDEI: 2 Syntypen, Türkei, Coll. v. Heyden.

rubella Broun, 1914, Somatidia

Bull. New Zealand Inst. 1, 254-255

12 Syntypen, New Zealand, Mount Hutt und Mc. Clennan's Bush, 1.500 ft., IV.1912, durch Hall.

SDEI: 1 Syntypus, New Zealand, Canterbury, Mt. Hult, Coll. Hall.

\section{rubricatum GOUNELLE, 1909, Ibidion}

Ann. Soc. ent. Fr. 77, 680-681

10 Syntypen, Brasilien, Prov. Goyaz, Jatahy.

SDEI: 1 Syntypus, Brésil, Prov. Goyaz, Jatahy.

rufa KraAtz, 1879, Pidonia

Dtsch. ent. Ztschr. 1879, 101-102

o Holotypus, Ost-Sibirien, Amur, leg. Christoph, im SDEI, Coll. KraATZ.

\section{rufa GounelLe, 1911, Sphecomorpha}

Ann. Soc. ent. Fr. 80, 49-50

$70^{\star}, 5$ ㅇ Syntypen, Brasilien, Prov. Goyaz, Jatahy.

SDEI: 2 Syntypen, mit den Angaben der Beschreibung.

ruficauda Gounelle, 1909, Haruspex

Ann. Soc. ent. Fr. 77, 654, Fig. 24

Mehrere Ex., Brasilien, Prov. Goayz, Jatahy; 1 Ex. Minas, Sertão de Diamantina; leg. GounElLe, Syntypen.

SDEI: 1 Syntypus, Brésil, Prov. Goyaz, Jatahy, Coll. KraATz.

\section{ruficornis Broun, 1914, Somatidia}

Bull. N. Zealand Inst. 1, 255-256

Mehrere Syntypen, New Zealand, Pudding Hill und Mc Clennan's Bush, nahe Methven, IV., V.1912, leg. Hall. SDEI: 1 Syntypus, New Zealand, Canterbury, Pudding Hill, Coll. Hall.

rufipenne cankiriense BrEunING, 1962, Dorcadion

Reichenbachia 1, 39

Holotypus, mehrere Paratypen, Anatolien, Cankiri, IV.1961, leg. Muche, in Coll. Muche.
SDEI: 3 Paratypen, Anatolien, Cankiri, IV., leg. Muche.

\section{rufobrunnea HEYDEN, 1894, Conizonia}

Dtsch. ent. Ztschr. 1894, 88

o Holotypus, Asia minor, Mardin (Kastomuni, nicht Mardin!), durch Preiss, in Coll. v. Heyden, im SDEI.

\section{rufogenuum ReITTER, 1895, Dorcadion}

Wien. ent. Ztg. 14, 161

Mehrere Syntypen, Thian-Schan, leg. HAusER.

SDEI: 6 Syntypen, Thian-Schan, Musart, VI.1894, Coll.

Hauser, Coll. Leonhard, Coll. F. Müller.

\section{rufoscapa Auriviluius, 1907, Sophronica}

Dtsch. ent. Ztschr. 1907, 81

Mehrere Syntypen, Kamerun, leg. ConRadT, im SDEI.

SDEI: 4 Syntypen, Kamerun, leg. ConRadT, Coll. KraATz.

\section{rufovittatus AuriviLlius, 1907, Calanthemis}

Dtsch. ent. Ztschr. 1907, 78

Mehrere Syntypen, Kamerun, leg. ConRadT, via SchenKLING an AURIVILLIUS, im SDEI.

SDEI: 1 Syntypus, Kamerun, leg. ConradT, Coll. KraAtZ.

rufus var. geniculatus KRAATZ, 1863, Stenopterus

Berl. ent. Ztschr. 7, 103

Syntypen, Griechenland.

SDEI: Lectotypus, Graecia; 1 Paralectotypus, Attica, Designation: SAMA.

rusticum GOUNELLE, 1909, Ibidion

Ann. Soc. ent. Fr. 77, 682-683

Mehrere Syntypen, Brasilien, Prov. Goyaz, Jatahy.

SDEI: 1 Syntypus, Brésil, Prov. Goyaz, Jatahy, Coll. KraAtz.

salicicola StILLER, 1934, Caenoptera

Mitt. Dtsch. Ent. Ges. 5, 37-38

Mehrere Syntypen, Ungarn, Szeged.

SDEI: 6 Syntypen, Hungaria, Szeged, leg. STILLER.

salomonum BreUnING, 1970, Scituloglaucytes

Bull. Soc. Ent. Mulhouse 26, Fig. 7

Holotypus, 7 Paratypen, Salomones, Insel Tulagi, im Museum Paris und SDEI.

SDEI: 3 Paratypen, engl. Salomons, Ins. Tulagi.

\section{santacrusis HELLER, 1935, Glaucites}

Arb. morph. tax. Ent. 2, 267-268

Mehrere Syntypen, Santa-Cruz, Vanikoro, leg. MaLcher, im Museum Dresden und SDEI.

SDEI: 1 Syntypus, Sta Cruz I., Vanikoro, leg. MALCher.

sapkaianum KRÄTSCHMER, 1987, Dorcadion

Ent. Ztschr. 97, 337-339

$\sigma^{\star}$ Holotypus, Paratypen, Griechenland (Thraki), Sapka, 1000 m, 4.IV.1986, leg. KRÄtschmer, in Coll. 
Krätschmer. Paratypen vom selben Fundort, in Coll. Holzschuh, Drovnik und KRäTschmer.

SDEI: 2 Paratypen, G R Alexandropolis, Sapka, 1300 m, 4.IV.1986, leg. DrovNik.

sarda GounELLE, 1912, Tamenes

Bull. Soc. ent. Fr. 1912, 133-135, Fig. 1

Holotypus, Amérique centrale, Chiriqui, im SDEI.

sareptanum KRAATZ, 1873, Dorcadion

In: KüsteR, Käf. Europ. 29, 74

Mehrere Syntypen; Sarepta, leg. BECKER; bei Astrachan. SDEI: 3 Syntypen, Sarepta, Coll. KraATz.

sauteri SCHWARZER, 1925, Glenea

Ent. Bl. 21, 150

Mehrere Syntypen, Formosa; Fuhosho, 7.VIII.; Alikang, 7.X.; Kosempo, VI.1909/ V.1912; leg. SAUter.

SDEI: Formosa, leg. SAuter, Syntypen: 3 Ex. Kosempo, V., 22.VI.1912; 2 Ex. Fuhosho, 7.VIII.; 1 Ex. Alikang, 7.X.

sauteri LAMEERE, 1913, Megopis

Arch. Naturg. 79 A, Heft 7, 175-176

o Holotypus, Formosa, Kosempo, VI., leg. Sauter, im SDEI 1912.

scabricolle caramanicum DanIEL, 1903, Dorcadion

Münch. Kol. Ztschr. 1, 332

Mehrere Syntypen, Kleinasien, bei Bulghar-Maaden und Bulghar-Magara, E. V., leg. v. BoDEMEYER.

SDEI: 2 Syntypen, Asia minor, Bulghar-Maaden, Bulghar-Magara, leg. v. Bodemeyer, durch LeONHARD, Coll. KraAtz.

scabrosum GounelLe, 1909, Gnomidolon

Ann. Soc. ent. Fr. 77, 663-664

13 Syntypen, Brasilien, Prov. Goyaz, Jatahy.

SDEI: 1 Syntypus, Brésil, Prov. Goyaz, Jatahy, Coll. KrAATZ.

scabrum KraAtz, 1882, Callidium

Dtsch. ent. Ztschr. 26, 115

Holotypus, Margelan, leg. Haberhauer, in Coll. KraAtz. SDEI: Holotypus, Namangan.

scalaris occidentalis SCHMIDT, 1922, Anubis

Arch. Naturg. 88, A 6, 141

Holotypus, Angola, Benguella, leg. Wellmann, im SDEI, Coll. KraAtz.

schenklingi LAMEERE, 1912, Aulacopus

Mém. Soc. ent. Belg. 21, 140

đ Holotypus, Uganda, im SDEI, Britisch Uganda, leg. Grauer.

schultzei HEYDEN, 1894, Dorcadion

Dtsch. ent. Ztschr. 1894, 86 $20^{\star}, 1$ ㅇ Syntypen, Asia minor, Mardin, in Coll. v. HeyDEN und Coll. Preiss.

SDEI: 2 Syntypen, Mardin, durch STAudinger, Coll. v. Heyden.

schwarzeri HeLLeR, 1926, Heterotaxalus

Tijdschr. Ent. 69, 42-43, Taf. 5, Fig. 4

Mehrere Syntypen, Nova Guinea Germanica, leg. WAHNES, ex Coll. F. Müller, im Museum Dresden und SDEI.

SDEI: 7 Syntypen, D. Neu-Guinea, leg. WAHnES, durch FrankLin MüLLER.

scotodes continentalis PLAVILSTSHIKOV, 1936, Leptura

Faune de URS 21, 371-372, 564, Fig. 184, 185

Mehrere Syntypen, südl. Primorje (weitere Angaben siehe Originalbeschreibung), leg. Plavilstshikov.

SDEI: 1 ơ Syntypus, Ussuri, Ossinovka, 20.VIII.1917, leg.

ELSKY.

\section{scrobicolle KRAATZ, 1873, Dorcadion}

In: Küster, Käf. Europ. 29, 97

Mehrere Syntypen, Kleinasien, in Coll. Mniszech und Coll. KraAtz.

SDEI: 1 Syntypus, Asia minor, Coll. KraATz.

\section{scutellata SCHWARzER, 1925, Dorcadion}

Ent. Bl. 21, 66

Mehrere Syntypen, Formosa; Kosempo, 7.XII.1909/ X.1911; Alikang, 22.IX.; Kankau, VI.1912; leg. SAUTER.

SDEI: Formosa, leg. SAuter, Syntypen: 1 Ex. Kankau, Koshun, VI.1912; 1 Ex. Alikang, 22.IX.; 1 Ex. Kosempo, X.1911.

\section{seabrai DIAS, 1988, Mysteria}

Rev. Bras. Ent. 32, 144-145, Fig. 16, 31, 49, 66, 80, 81

$0^{\star}$ Holotypus, Argentinien, La Gallareta, I.1907, im Mus.

La Plata; Paratypen siehe Originalbeschreibung.

SDEI: $1 \sigma^{\top}$ Paratypus, Esperanza (Argentina).

sellaemontis HeLleR, 1912, Pseudaelara

Ent. Mitt. 1, 176, Fig. 5

Mehrere Syntypen, Nova Guinea Germanica, Sattelberg, Coll. v. BenNigsen.

SDEI: 2 Syntypen, N. Guinea, Sattelberg, leg. v. BENNIGSEN, Coll. v. BenNigsen.

\section{semenovi Heyrovsky, 1934, Moechotypa}

„asop. Cs. Spol. Entom. 31, 172-173

15 Syntypen, Giufu-Shan, 1500-2000 m, Szechuan, in Coll. Reitter und Coll. Heyrovsky.

SDEI: 2 Syntypen, Szechuan, Giufu-Shan, 1500-2000 m, leg. REITTER.

semenovi kuvakense DANILEVSKY, 2002, Dorcadion Les Cahiers Magellanes 10(2001): 16 (kuvakensis lapsus) ○ Holotypus, Kirgizia, East Kirgizky Ridge, Kuvaki Pass, 2300 m, 20.V.1998, leg. S. Toropov, Coll. Danilevsky; 
152 Paratypen vom gleichen Fundort in der Sammlung des Autors.

SDEI: $1 \sigma^{\star}$ Paratypus, Kirgizia, Kuvaky Pass, 2000 m, 14.IV.2000, leg. M. DANILEVSKY.

semilucens KraAtz, 1873, Dorcadion

In: Küster, Käf. Europ. 29, 84

Mehrere Syntypen, Mongolei, durch Kindermann, in Coll. Mniszech, Coll. KraAtz.

SDEI: 1 Syntypus, Caucasus, durch Mniszech.

seminudum KrAAtz, 1873, Dorcadion

In: Küster, Käf. Europ. 29, 83

Mehrere Syntypen, Kaukasus, in Coll. Mniszech und Coll. KraATz.

SDEI: 2 Syntypen, Kaukasus, durch Mniszech, Coll. KraAtz.

semipubescens GounelLE, 1911, Smodicum

Ann. Soc. ent. Fr. 80, 127-129

$10^{\star}, 1$ ㅇ , Peru, Vilcanota; $10^{\star}, 1$ 우, Ocobambe, im SDEI: Syntypen.

SDEI: $10^{\wedge}, 1$ ㅇ Paralectotypen, Peru, Ocobama, Coll. KraAtZ, Designation: Martins 1973.

semirufa KRAATZ, 1882, Oberea

Dtsch. ent. Ztschr. 26, 115-116

Mehrere Syntypen, um Margelan.

SDEI: 1 Syntypus, Margelan, Coll. KraATz.

semirufus KrAATZ, 1884, Cleroclytus

Dtsch. ent. Ztschr. 28, 225-226

Holotypus, Turkestan, Osch, durch Staudinger, im SDEI, Coll. KraATz.

\section{semivelutinum SCHWARzER, 1925, Aphrodisium}

Ent. Bl. 21, 24-25

Holotypus, Formosa, Hoozan, IX.1910, leg. SAUTER, im SDEI.

\section{semivelutinum KrAATZ, 1873, Dorcadion}

In: Küster, Käf. Europ. 29, 82

Mehrere Syntypen, Kleinasien, durch Mniszech.

SDEI: 2 Syntypen, ohne Fundort, Coll. KraATz.

sericatula GounelLe, 1909, Appula

Ann. Soc. ent. Fr. 77, 649-650, Fig. 23

Mehrere Syntypen, Brasilien, Prov. Goyaz, Jatahy, São Paulo, Minas, leg. GounelLE.

SDEI: 2 Syntypen, Brésil, Et. de Sao Paulo, Cerqueira Cesar, Coll. Vogel, Coll. KraAtz.

\section{sericatulum KraAtZ, 1873, Dorcadion}

In: Küster, Käf. Europ. 29, 98

Mehrere Syntypen, Caucasus, durch Heyne, Coll. MnisZECH

SDEI: 2 Syntypen, Caucasus, durch Heyne, Coll. MnIsZECH-Coll. KraATZ. setigera SCHWARzER, 1925, Cleomenida

Ent. Bl. 21, 29-30

Mehrere Syntypen, Formosa: Kosempo, 7.VII.1911/ 22.V. 1912/ 7.VI.1912; Kankau, V.1912; Sokutsu, 7.VI.1912; leg. SAUTER.

SDEI: Formosa, leg. SAUTER, Syntypen: 13 Ex. Kosempo, V., 22.V., 7.VI.1912/ 7.VII.1911/ 7.XII.; 2 Ex. Kankau, Koshun, V., VI.1912; 2 Ex. Sokutsu, 7.VI.1912.

sexplagiatus Melzer, 1927, Atelographus

Rev. Mus. Paul. 15, pt 1, 578

Mehrere Syntypen, Brasil, Annaburg, Estado de Santa Catharina, leg. Schmith.

SDEI: 3 Syntypen, Est. S. Catharina, Joinville, X.1921, leg. SCHMith, ded. Melzer.

sicula GANGLBAUER, 1885, Leptura

Verh. Zool. Bot. Ges. Wien 35, 515-516

2 Syntypen, Sicilia, im Museum Wien und via StIERLIN an GANGLBAUER.

SDEI: 1 Syntypus, Sicilia, Coll. STIERLIN.

siculum Stierlin, 1864, Callidium

Berl. ent. Ztschr. 8, 152

Mehrere Syntypen, Sicilien.

SDEI: 2 Syntypen, Sicilien, Coll. STIERLIN.

signaticornis SCHWARzER, 1925, Linda

Ent. Bl. 21, 154

Mehrere Syntypen, Formosa: Kosempo, V.1912; Kankau, VII.1912; Sokutsu, 7.VI.1912; leg. SAUTER.

SDEI: Formosa, leg. SAUTER, Syntypen: 1 Ex. Kankau, Koshun, VII.1912; 2 Ex. Sokutsu, Banshoryo-Distr., 7.VI., 7.VII.1912.

signaticornis SCHWARzER, 1925, Serixia

Ent. B1. 21, 149

Mehrere Syntypen, Formosa, Kankau, IV., V.1912, leg. SAUTER.

SDEI: 3 Syntypen, Formosa, Kankau, Koshun, 7.IV., IV., V.1912, leg. SAUTER.

signaticornis MeLzeR, 1920, Temnopis

Rev. Mus. Paul. 12, 421-423, Fig. 3, 4

24 Syntypen, Brasil, Passa Quatro, Sul de Minas, in Coll. Zikan, im Museum Paulista und in Coll. Melzer.

SDEI: 1 Syntypus, Brasil, Minas Passa Quatro, I.1918, leg. CAMPos.

signatus SCHWARzER, 1925, Thranius

Ent. Bl. 21, 23-24

Mehrere Syntypen, Formosa: Fuhosho, 7.IX.; Kosempo, 7.IX.1911/ VI.1912; leg. SAUTER.

SDEI: Formosa, leg. SAuter, Syntypen: 1 Ex. Fuhosho, 7.IX.; 2 Ex. Kosempo, 7.IX.1911/ VI.1912. 
silacea AuriviluIus, 1907, Phryneta

Dtsch. ent. Ztschr. 1907, 80

o Holotypus, Togo-Amedzowe, im SDEI, Coll. KraATz.

simile KraAtz, 1884, Dorcadion

Dtsch. ent. Ztschr. 28, 234

` Holotypus, südliches Kleinasien, Malatia, im SDEI, Coll. KraAtz.

simillimum Melzer, 1932, Alcidion

Rev. Ent. S. Paulo 2, 224-225, Taf. 3, Fig. 6

Brasilien, Estado de São Paulo, Bosque da Saude perto da Capital, leg. Melzer; Alto da Serra, leg. Ohaus; Est. Sta. Catharina, Joinville, leg. Schmith; Hansa-Humboldt, leg. Maller; in Coll. Melzer; Holotypus, mehrere Paratypen. SDEI: 1 Paratypus, Est. S. Catharina, Joinville, I.1924, leg. SCHMITH.

\section{similis KRAATZ, 1879, Grammoptera}

Dtsch. ent. Ztschr. 23, 102

1 ơ $^{*} 2$ + Syntypen, Ost-Sibirien, Amur, leg. Christoph. SDEI: 3 Syntypen, Amur, Coll. KRAATz.

simplarium HeYDEN, 1885, Callidium

Dtsch. ent. Ztschr. 29, 296-297

Mehrere Syntypen, Turkestan, Namagan, leg. STAudinGER.

SDEI: 3 Syntypen, mit den Angaben der Beschreibung, 1885, Coll. v. Heyden.

\section{simplicipes Gounelle, 1911, Coremia}

Ann. Soc. ent. Fr. 80, 81-82, Fig.

1 ㅇ, Brasilien, Prov. Goyaz, Jatahy; 1 ơ, Bahia, Umgebung von Condeuba; 4 Ex. N. W. Argentinien, leg. Steinbach, im SDEI: Syntypen.

SDEI: 4 Syntypen, N. W. Argentinien, leg. Steinbach, Coll. KraAtz.

simplonica StIERLIN, 1880, Leptura

Mitt. Schweiz. ent. Ges. 5, 550-551

Holotypus, Simplon, im SDEI, Coll. STIERLIN.

singularis KRAATZ, 1879, Brachyclytus

Dtsch. ent. Ztschr. 23, 107-108, Taf. 1, Fig. 6

Holotypus, Ost-Sibirien, Amur, leg. CHRIstoph, im SDEI 1877.

\section{sodale HAMPE, 1852, Docradion}

In: WAGNER, Reise n. Persien 2, 313

Mehrere Syntypen, Kaukasus, Trans-Kaukasien, Armenien, Kurdistan und West-Persien.

SDEI: 1 Syntypus, ohne Fundort, Coll. KraATz.

solskyi KraATz, 1879, Pachyta

Dtsch. ent. Ztschr. 23, 71-72, 76, Taf. 1, Fig. 15

2 Syntypen, Ost-Sibirien, Baikal-See, durch v. Solsky, Coll. KraAtz.

SDEI: 1 Syntypus, Baikal, durch v. Solsky, Coll. KraAtz. sormeoides AuRIVILLIUS, 1908, Heteroc/ytomorpha

Dtsch. ent. Ztschr. 1908, 219-220, Fig. 11, 11a

Mehrere Syntypen, Neu-Pommern, Gazelle-Halbinsel, leg. v. BenNigsen.

SDEI: 1 Syntypus, Gazelle-H.-Insel, Coll. v. Bennigsen.

soror KRAATZ, 1882, Agapanthia

Dtsch. ent. Ztschr. 26, 336-337

Mehrere Syntypen, Samarkand, leg. Haberhauer.

SDEI: 1 Syntypus, ohne Fundort, Coll. KraATz.

sparsus ReItTER, 1886, Clytus

Dtsch. ent. Ztschr. 30, 67

Mehrere Syntypen, im Lycischen Taurus, Adalia.

SDEI: 3 Syntypen, Adalia.

starcki ReitTeR, 1888, Cortodera

Wien. ent. Ztg. 7, 280; als var. von alpina MÉNÉTRIEs beschrieben Mehrere Syntypen, Abago, leg. Starck.

SDEI: 3 Syntypen, Caucasus occid., Abago, leg. STARck, Coll. STIERLin.

stenostola KraAtz, 1879, Asaperda

Dtsch. ent. Ztschr. 23, 227-228, Taf. 2, Fig. 14, 14a

2 Syntypen, Ost-Sibirien, Amur, leg. Christoph, in Coll.

KrAATZ, im SDEI 1877.

sterni KraAtZ, 1870, Clytus

Berl. ent. Ztschr. 14, 219, Taf. 3, Fig. 1

Mehrere Syntypen, aus Waldholz (zur Insektenzucht) von STERN in Frankfurt a. M., im Frühsommer 1868 gezogen, via Stern an KraAtZ.

SDEI: 4 Syntypen, Frankfurt a. M., Coll. STERN.

striolatum KraATZ, 1873, Dorcadion

In: KüsteR, Käf. Europ. 29, 93

Mehrere Syntypen, Türkei, durch Heynemann, Sallé, Mniszech, Küster und KraAtz.

SDEI: 2 Syntypen, Kaukasus, Coll. KraATz.

subfasciata SchWARzer, 1925, Miaenia

Ent. Bl. 21, 148

Mehrere Syntypen, Formosa, Sokutsu, 22.VI.1912, leg. SAUTER.

SDEI: 1 Syntypus, Banshoryo Distr., Sokutsu, 1912, leg. SAUTER.

\section{subuniforme ScHWARzER, 1925, Ceresium?}

Ent. Bl. 21, 22

Mehrere Syntypen, Formosa, Kosempo, 7.VI.1912, leg. SAUTER.

SDEI: 2 Syntypen, Formosa, Kosempo, 7.IX.1909/ 22.V. 1912, leg. SAUTER.

sulcipenne KüSTER, 1847, Dorcadion

In: Küster, Käf. Europ. 8, 87-88

Mehrere Syntypen, Caucasus und Türkei.

SDEI: 1 Syntypus, ohne Fundort, Coll. KraAtz. 
sumatrana NonfRIED, 1894, Merionoeda

Dtsch. ent. Ztschr. 1894, 206-207

Mehrere Syntypen, N. O. Sumatra, Tebing-Tinggi, leg. SCHultheiss.

SDEI: 5 Syntypen, mit den Angaben der Beschreibung, 8.IX.1884, Coll. Schultheiss.

\section{sumatrensis SCHWARZER, 1924, Distenia}

\section{Treubia 5, 236}

Mehrere Syntypen, Süd-Sumatra, Wai Lima, Lampongs, XI.-XII.1921, leg. KARNY \& SiEBERs.

SDEI: 1 Syntypus, mit den Angaben der Beschreibung, No. 17, ex Museum Buitenzorg.

\section{superbum AuRIVILlius, 1908, Chloridolum}

Dtsch. ent. Ztschr. 1908, 212

Mehrere Syntypen, Salomon-Inseln, leg. v. Bennigsen. SDEI: 2 Syntypen, D. N.-Guinea, Salomon-Inseln, Coll. KRAATZ.

suturale AuriviLLIUS, 1908, Hypatium

Dtsch. ent. Ztschr. 1908, 211-212, Taf. 3, Fig. 2

Mehrere Syntypen, Westafrika, Benguella, Coll. v. BENNIGSEN.

SDEI: 1 Syntypus, W. A., Benguella, Coll. v. BennigSEN.

suvorovi konyrolenum DANILEVSKY, 1996, Dorcadion Lambillionea 96: 458-459 (konyrolenus lapsus)

o Holotypus, 201 Paratypen, Kzakhstan, Konyrolen S, Dzhungarsky Alatau, 1250 m, 8.V.1994, leg. M. DANILEVSKY.

SDEI: $1 \sigma^{\star}$ Paratypus, vom gleichen Fundort.

\section{taihorensis SchWARzER, 1925, Chlorophorus}

Ent. Bl. 21, 27; als var. von signaticollis CASTelnau \& GoRY beschrieben

Mehrere Syntypen, Formosa, Taihorin, 7.VI.1911, leg. SAUTER.

SDEI: 2 Syntypen, mit den Angaben der Beschreibung.

\section{tenebrica Broun, 1893, Somatidia}

Man. N. Zealand Col. 7, 1500

20 Syntypen, New Zealand, Maketu, Hunua Range.

SDEI: 4 Syntypen, Auckland, Maketu, Hunua, Coll. BROUN.

tenebrosus VILLIERS, 1980, Nethinius

Faun. Madagasc. 52, 43, 99, Fig. 267-269

○ Holotypus, Madagascar, Baie d'Antongil, 98, leg. Mocquerys, im Museum Paris; $1 \sigma^{*}, 2$ o Paratypen, dito, im Museum Paris, Museum Brüssel und Museum Berlin.

SDEI: 1 Paratypus, Madagascar, Coll. KraATz.

tenuicorne KraAtz, 1879, Asenum

Dtsch. ent. Ztschr. 23, 97 nota
1 ơ $^{\star}, 1$ i Syntypen, Krim, leg. Ribbe.

SDEI: Lectotypus, 1 Paralectotypus, Krim, Coll. KraAtz, Designation: SAMA.

tesserula var. impunctata HEYDEN, 1877, Leptura

Dtsch. ent. Ztschr. 21, 420

1 Syntypus, Siebenbürgen, in Coll. Heyden; 1 Syntypus, Caucasus, in Coll. TüRk.

SDEI: Lectotypus, Transylv., STEnz, Designation: SAmA.

tianshanskii Suvorov, 1910, Compsodorcadion

Rev. Russe d'Ent. 10, 67-69

Mehrere Syntypen, im Semirjetshje-Gebiet.

SDEI: 1 Syntypus, Semirjetshje, Tshulak, Coll. KL ̈̈GER.

tonsa DANIEL, 1891, Leptura

Col. Stud. 1, 31-32

Mehrere Ex. Georgien, Helenendorf, im Wiener Hofmuseum; Transkaukasich-persisches Grenzgebiet, AraxesThal, Coll. Reitter; 2 Ex. Cilicischer Taurus, Külek, in Coll. v. Heyden und Coll. nostr.; 1 Ex. aus Beyrut; 2 Ex. Hyrcanien, in Coll. Staudinger: Syntypen.

SDEI: 1 o $^{\star}$ Paralectotypus, Külek, leg. Korb, Coll. v. HeYDEN, Designation: SAMA.

\section{tricolor GOUNELLE, 1909, Stizocera}

Ann. Soc. ent. Fr. 77, 644-645, Fig. 22

Mehrere Syntypen; Brasilien, Prov. Goyaz, Jatahy; Minas,

Bahia; leg. GounelLe.

SDEI: 2 Syntypen, Prov. Bahia, Coll. KraAtz.

trilineata HINTZ, 1913, Crossotofera

Dtsch. ent. Ztschr. 1913, 202

Mehrere Syntypen, Congo Belge, Kondué, Kamerun, Bipindi.

SDEI: 1 Syntypus, Congo Belge, Kondué, leg. LujA, Coll. KRAATZ.

tripartita HEYDEN, 1889, Leptura

Dtsch. ent. Ztschr. 1889, 329

+ Holotypus, Syria, via Staudinger an v. Heyden, im SDEI.

trisignata var. maroccana HEYDEN, 1886, Leptura Dtsch. ent. Ztschr. 30, 85; nom. nov. pro L. oblongomaculata var. tangeriana Heyden: Dtsch. ent. Ztschr. 25, 252; 1881. Syntypen, Tanger.

SDEI: Lectotypus, Marocco, Deyrolle, in Coll. Heyden, Designation: SAMA.

tristicula KrAATz, 1879, Pseudopidonia

Dtsch. ent. Ztschr. 23, 104; als var. von alticollis KRAATZ beschrieben.

Holotypus, Ost-Sibirien, Amur, leg. Christoph, im SDEI 1877, Coll. KraATZ. 
tristiculus KraAtz, 1879, Pogonocherus

Dtsch. ent. Ztschr. 23, 115

Holotypus, Ost-Sibirien, Amur, leg. CHrIstoph, im SDEI (ohne Fundort).

\section{tristriatum Suvorov, 1913, Dorcadion}

Rev. Russe d'Ent. 13, 71-72

Mehrere Syntypen, Terek-Gebiet, Umgebung von Kislowodsk, E. IV. - A.V.1894, leg. Suvorov.

SDEI: 3 Syntypen, mit den Angaben der Beschreibung, 5.V.1894, Coll. KLÄGER.

\section{truncatipennis Melzer, 1934, Adetus}

Rev. Ent. Rio de Janeiro 4, 81, Taf. 1, Fig. 4

Holotypus, 1 Paratypus, Rio de Janeiro, Manguinhos, leg. FIscher; Rio Grande do Sul, Alto Feliz, leg. Buck; in Coll. Melzer und im SDEI.

SDEI: 1 Paratypus, Ctr. Brasilien, Rio de Janeiro, 22.V. 1918, leg. FisCHER.

\section{tuberculata Nonfried, 1894, Stesilea}

Dtsch. ent. Ztschr. 1894, 210-211

Mehrere Syntypen, N. O. Sumatra, Tebing-Tinggi, leg. SCHULTHEISs.

SDEI: 1 Syntypus, mit den Angaben der Beschreibung.

tuerki GanglBaUER, 1884, Dorcadion

Verh. Zool. Bot. Ges. Wien 33 (1883), 486

Mehrere Syntypen, Persien, Hadschgabad, leg. Lederer, durch KraATZ und Türk.

SDEI: 1 Syntypus, Hadschgabad, leg. Lederer, Coll. KRAATZ.

\section{turcestanicum KraAtZ, 1881, Dorcadion}

Dtsch. ent. Ztschr. 25, 335

Mehrere Syntypen, Turkestan, Margelan.

SDEI: 1 Syntypus, Turkestan, Coll. KraATz.

turcestanicus HeYDEN, 1886, Stromatium

Dtsch. ent. Ztschr. 30, 193

+ Holotypus, Turkestan, via Koltze an v. Heyden, im SDEI (ohne Fundort, Nr. 98).

ugandensis MARTINS, 1980, Xystrocera

Pap. Avuls. Zool. S. Paulo 33 (5), 108-109, Fig. 32

o Holotypus, Uganda, leg. Grauer, Coll. v. Bennigsen, im SDEI.

umbripennis ReITTER, 1890, Cartodera

Wien. ent. Ztg. 9, 245

Mehrere Syntypen, Araxesthal bei Ordubad.

SDEI: 1 Syntypus, Caucasus, Araxesthal, leg. LederReitter, Coll. Stierlin.

\section{umtaliensis SCHMIDT, 1922, Anubis}

Arch. Naturg. 88, A 6, 140

Mehrere Syntypen, Rhodesia, Umtali, leg. Bodong, im Museum Berlin und SDEI.
SDEI: 5 Syntypen, mit den Angaben der Beschreibung, Coll. Kratitz.

uncinatum GounelLe, 1909, Hexoplon

Ann. Soc. ent. Fr. 77, 659-660

Mehrere Syntypen, Brasilien, Prov. Goyaz, Jatahy.

SDEI: 2 Syntypen, Brésil, Prov. Goyaz, Jatahy, Coll. KraAtZ.

uniformis HINTZ, 1913, Pinacosternodes

Dtsch. ent. Ztschr. 1913, 199

Mehrere Syntypen, Congo Belge, Kondué.

SDEI: 1 Syntypus, Congo-Belge, Kondué, leg. Luja, Coll. KraAtZ.

urdzharicum PLAVILSTSHIKOV, 1937, Dorcadion

Acta Ent. Mus. Nat. Prague 15, 29-30, 33

Mehrere Syntypen, Kasachstan bor., Urdzhar, 16.-25.V. 1934, in Coll. du Filial de Kasachstan de l'Académie des Sciences de l'UKSS und in Coll. Plavilstshikov. SDEI: 1 o $^{\top}$ Syntypus, Dzhungaria, cass. bor., Urdzhar.

urundiensis Quentin \& VILLIERS, 1970, Dorcasomus

Ann. Soc. ent. Fr. (N. S.) 6 (1), 32, Fig. 7, 21

+ Holotypus, Burundi, N. Urundi, leg. Grauer, im SDEI.

uvarovi Suvorov, 1911, Compsodorcadion

Rev. Russe d'Ent. 11, 279-280, 282

Mehrere Syntypen, Uralsk-Gebiet, Umgebung der Stadt Temir, 1.-2.IV.1908, leg. Uvarov und Borodin.

SDEI: 3 Syntypen, Sibirien occ. mer., Temir, Uralsk, Knigul-Kul, 16., 23.IV., 3.V.1908, leg. Uvarov und Borodin, Coll. KLÄGER.

vadoni VILLIERS, 1980, Nethinius

Faun. Madagascar 52, 40, 76-78, Fig. 212-217

○ Holotypus, Madagascar, région de Maroantsetra, XII. 1934, leg. VAdon, im Museum Paris, Coll. VAdon; mehrere Paratypen, siehe Originalbeschreibung.

SDEI: 1 Paratypus, Madagascar, Coll. KraATz.

\section{vanikorana HELLER, 1935, Glenea}

Arb. morph. tax. Ent. 2, 268

Mehrere Syntypen, Santa-Cruz, Vanikoro, im Museum Dresden und SDEI.

SDEI: 1 Syntypus, Sta Cruz I., Vanikoro, leg. MALCHER.

variabile GounelLe, 1909, Protosphaerion

Ann. Soc. ent. Fr. 77, 635, Fig. 20

Mehrere Syntypen, Brasilien, Prov. Goyaz, Jatahy; Minas, Serra do Caraca, Serra et Sertao de Diamantina, Sao Paulo, vallée du Rio Pardo, Pernambuca, Pery-Pery, leg. GOUNELLE.

SDEI: 2 Syntypen, Brésil, Prov. Goyaz, Jatahy, Coll. KRAATZ. 
variabilis SchWARzER, 1925, Mesopenthea

Ent. Bl. 21, 67-68

Mehrere Syntypen, Formosa, Sokutsu, 7.VI.1912, leg. SAUTER.

SDEI: 2 Syntypen, Sokutsu, Banshoryo Distr., 7.VI.1912, leg. SAUTER.

\section{varicans GOUNELLE, 1909, Gnomidolon}

Ann. Soc. ent. Fr. 77, 662

Mehrere Syntypen, Brasilien, Prov. Goyaz, Jatahy, Bahia, Minas, São Paulo.

SDEI: 1 Syntypus, Brasilien, Prov. Goyaz, Jatahy, Coll. KRAATZ.

variegata AuRIVILLIUS, 1907, Hyllisia

Dtsch. ent. Ztschr. 1907, 82

Mehrere Syntypen, Togo, leg. ConRadT, im SDEI.

SDEI: 1 Syntypus, Togo, leg. Conradt, Coll. KraAtz.

variegatum GANGlBaUeR, 1884, Dorcadion

Verh. Zool. Bot. Ges. Wien 33 (1883), 502-503

SDEI: $10^{\star}, 1$ \% Syntypen, Syrien, leg. STAudinger, Coll. KRAATZ.

\section{variepennis Schwarzer, 1925, Camptomine?}

Ent. Bl. 21, 147-148

Mehrere Syntypen, Formosa, Kankau, VI.1912, leg. SAUTER.

SDEI: 1 Syntypus, Formosa, Kankau, Koshun, VI.1912, leg. SAuter.

veneficella Martins, 1976, Gorybia

Arq. Zool. S. Paulo, 27 (3/4), 307-308, Fig. 166

o Holotypus, Brasil, Mato Crosso, Chapada dos Guimarães, X., im National Museum Natur. Hist. Washington; Paratypen, siehe Originalbeschreibung.

SDEI: 1 Paratypus, Brésil, Prov. Goyas, Jatahy, Coll. KraAtZ.

verbasci var. viridicollis KRAATz, 1870, Clytus

Berl. ent. Ztschr. 14, 410, Taf. 3, Fig. 4a

2 Syntypen, Griechenland, in Coll. KraAtz.

SDEI: Lectotypus, Graecia, Designation: SAMA.

vicinus Melzer, 1931, Ozineus

Arch. Inst. Biol. S. Paulo 4, 68, Taf. 14, Fig. 21

Mehrere Syntypen, Bosque da Saude perto da Capital do Estado de São Paulo, in Coll. Melzer.

SDEI: 1 Syntypus, Brasilien, S. Paulo, Est. S. Paulo, 6.XI. 1921, SAUDE.

villosa HEYDEN, 1876, Cortodera

Dtsch. ent. Ztschr. 20, 318

o Holotypus, Hungaria, DAHL.

SDEI: Holotypus, Hung. DAHL, Nr. 346, in Coll. Heyden.

vinculatus HeLleR, 1914, Tmesisternus

Dtsch. ent. Ztschr. 1914, 316-317, Taf. 12, Fig. 10
Mehrere Syntypen, Nova Guinea Germanica, Kani, (recte: kant?) montes, im Museum Dresden und SDEI.

SDEI: 1 Syntypus, D. N. Guinea, Kani Geb., Coll. v. BENNIGSEN.

virens amphipatris SCHMIDT, 1922, Philematium

Arch. Naturg. 88, A 6, 105

1 Ex. Br. O. Afrika, Luitpoldkette, im SDEI; 1 Ex. Togo, Amedzowe, leg. Hauser (dieses Ex. stammt vielleicht von Ikutha, Fundortzettel wahrscheinlich verwechselt!); Syntypen.

SDEI: 1 Syntypus, Afrika or., Luitpoldkette, Coll. KraAtZ.

viridicincta AuriviluIus, 1907, Syndere

Dtsch. ent. Ztschr. 1907, 79

Mehrere Syntypen, Dahomey, via SchenkLING an AurIVILLIUS, im SDEI.

SDEI: 1 Syntypus, Dahomey, ex. Coll. Schneider, Coll. KRAATZ.

vittata AuriviLLIUS, 1907, Ocularia

Dtsch. ent. Ztschr. 1907, 79-80

Mehrere Syntypen, Kamerun, leg. ConRadt, via SchenKLING an AURIVILLIUS, im SDEI.

SDEI: 1 Syntypus, Kamerun, leg. ConradT, Coll. KRAATZ.

volgensis KraAtz, 1883, Phytoecia

Wien. ent. Ztg. 2, 276

Mehrere Syntypen, Wolga, durch Kindermann.

SDEI: 1 Syntypus, ohne Fundort, durch KInDERMAnN, Coll. KraAtz.

wewalkai HolzSCHuH, 1969, Chlorophorus

Arbgem. öst. Ent. 21, 77-78, Abb. 1

o Holotypus, 18 Paratypen, Türkei, Kizilcahaman, in Coll. Holzschun und leg. Wewalka, in Coll. Wewalka. SDEI: 1 Paratypus, Anatolien, Kizilcahaman, 13.-16.VI. 1968, leg. Holzschun.

\section{wickhami rhodopus LINSLEY, 1955, Crossidius}

Pan-Pacif. Ent. 31, 64-65

o Holotypus, California, Inyo Counly, Convict Lake, 15.IX.1954, leg. MacKenzie; Paratypen, siehe Originalbeschreibung.

SDEI: 1 Paratypus, California, Mammoth, 27.VIII.1946, leg. MacKenzie.

woltersi BRuCH, 1925, Prionapterus

Physis. B. Aires 8, 207-209, Fig. 8, 10

10 주 우 Syntypen: Argentinien, Prov. Catamarca, Westabhang des Anconquija: Zarso, leg. Weiser; Arenal, leg. WOLTERS.

SDEI: $10^{\star}$ Paralectotypus, Sarso, 3200 m Designation: GAlileo (Rev. Bras. Ent. 31, 612; 1987). 
yapensis AuriviLluus, 1908, Prosoplus

Dtsch. ent. Ztschr. 1908, 223

Mehrere Syntypen, Insel Yap, leg. v. Bennigsen.

SDEI: 3 Syntypen, Yap, Coll. v. Bennigsen.

\section{zarcoi Schramm, 1910, Dorcadion}

Bol. Real. Soc. Esp. Hist. Nat. 10, 285-286

Mehrere Syntypen, Epila, Saragosse, durch Zarco.

SDEI: 19 Syntypen, Epila, Saragosse, Coll. KLÄger,

Coll. v. Heyden (4 Ex. als ab. curvilineatum Schramm bezettelt).

\section{Wahrscheinlich nicht beschriebene Arten}

amitina KoLBE, Nitocris

SDEI: Typen: 1 Ex. Pangani St., Coll. v. Bennigsen; 1 Ex. D. O. Afrika, Uhehe, Iringa, I.-III.1899, leg. GötzE, commut, Kgl. Museum Berlin.

biapicata KoLBE, Apomecyna

SDEI: 1 Typus, Usambara, Coll. v. Bennigsen.

bipunctata KoLBE, Eremomoecha

SDEI: 1 Typus, Usambara.

comaratus DILLON, Pseudonephelatus

SDEI: 1 Typus, W. Afrika.

curtulus SCHWARzeR, Tmesisternus

SDEI: 6 Typen, D. Neu-Guinea, leg. WAHNES, durch FRANKLIN MÜLLER.

elongatus Schwarzer, Cereopsius

SDEI: 1 Typus, S. Sumatra, Wai Lima, Lampongs, XI.XII.1921, No. 17, leg. Karny \& Siebers, ex Museum Buitenzorg.

flavovittata KoLBE, Perhippopsis

SDEI: 1 Typus, Usaramo, Coll. v. Bennigsen.

formosanus SCHWARZER, Coptops

SDEI: 3 Typen, Formosa, Paroe, Paiwan-Distr., 1912, leg. SAUTER.

franklinmuelleri SCHWARzER, Demonax

SDEI: 3 Typen, Queensland, leg. FrankLIn Müller.

franklinmuelleri SCHWARZER, Glenea

SDEI: 9 Typen, D. Neu-Guinea, leg. WAHNES, durch FrankLIN MÜLLER. fuscoplagiatus SCHWARZER, Dihammus

SDEI: 2 Typen, Nalanda, Weligama, 1899, leg. Horn.

janthipennis PIC, Astathes

SDEI: 5 Typen, Szechuan, Chungking, leg. ReITTER.

lata Schwarzer, Serixia

SDEI: 1 Typus, N. N. Guinea, Pionierbivak, VI.-VII.1920,

leg. v. Heurn, ex Museum Buitenzorg.

longicornis KoLBE, Stenopsis

SDEI: 1 Typus, Usambara, Coll. v. Bennigsen.

nebulosus Schwarzer, Anancylus

SDEI: 1 Typus, Malacka, leg. FrankLIn MülLER.

obliquefasciata KoLBE, Phryneta

SDEI: 1 Typus, Usambara, Coll. v. Bennigsen.

pallidipenne GounelLe, Bomarion

SDEI: 1 Typus, Brésil, Prov. Jatahy.

panganica KoLBE, Tragocephala

SDEI: 1 Typus, Pangani St., Coll. v. Bennigsen.

philomenus Dillon, Brunneochamus

SDEI: 1 Typus, Kamerun, leg. Conradt, Coll. KraAtz.

sansibaricus KoLBE, Taurotagus

SDEI: 2 Typen, Usambara, D. Togo.

sellatus KoLBE, Idactus

SDEI: 1 Typus, Pangani, Coll. v. Bennigsen.

solutus KoLBE, Bolbopterus

SDEI: 1 Typus, Usambara, Coll. v. Bennigsen.

spiniscapus Schwarzer, Osphryon

SDEI: 1 Typus, N. Guinea, Exped. Dormanpadbivak, X.1920, leg. v. Heurn, ex Museum Buitenzorg.

subchalybeata SCHWARzeR, Epania

SDEI: 1 Typus, Formosa, Kosempo, 7.VII.1911, leg. SAUTER.

vestita KoLBE, Sophronica

SDEI: 2 Typen, Dar-es-Salaam, leg. v. Bennigsen und Coll. v. Bennigsen. 